\title{
Systematic electronic-structure investigation of substitutional impurity diffusion and flux coupling in bec iron
}

\author{
Luca Messina, ${ }^{1, *}$ Maylise Nastar, ${ }^{2}$ Nils Sandberg, ${ }^{1,3}$ and Pär Olsson ${ }^{1}$ \\ ${ }^{1}$ KTH Royal Institute of Technology, Reactor Physics, SE-106 91 Stockholm, Sweden \\ ${ }^{2}$ DEN-Service de Recherches de Métallurgie Physique, CEA, Université Paris-Saclay, \\ F-91191 Gif-sur-Yvette, France \\ ${ }^{3}$ Swedish Radiation Safety Authority, Solna Strandväg 96, SE-171 16 Stockholm, Sweden \\ (Received 29 September 2015; revised manuscript received 6 April 2016; published 3 May 2016)
}

\begin{abstract}
The diffusion properties of a wide range of impurities (transition metals and $\mathrm{Al}, \mathrm{Si}$, and $\mathrm{P}$ ) in ferritic alloys are here investigated by means of a combined $a b$ initio-atomic diffusion theory approach. The flux-coupling mechanisms and the solute-diffusion coefficients are inferred from electronic-structure calculations of solutedefect interactions and microscopic jump frequencies. All properties except the second-nearest-neighbor binding energy are found to have a characteristic bell shape as a function of the $d$-band filling for the $4 d$ and $5 d$ series, and an M shape for the $3 d$ row because of the out-of-trend behavior of $\mathrm{Mn}$. The solute jump frequencies are governed by compressibility, which makes diffusion of large solutes faster, although this effect is partially compensated for by lower attempt frequencies and larger correlations with the vacancy. Diffusion coefficients are predicted in a wide temperature range, far below the experimentally accessible temperatures. In accordance with experiments, Co is found to be a slow diffuser in iron, and the same behavior is predicted for Re, Os, and Ir impurities. Finally, flux-coupling phenomena depend on the iron jump frequencies next to a solute atom, which are mainly controlled by similar electronic interactions to those determining the binding energies. Vacancy drag and solute enrichment at sinks systematically arise below a solute-dependent temperature threshold, directly correlated with the electronic-level interactions at the equilibrium and the saddle-point states. Early transition metals with repulsive second-nearest-neighbor interactions also diffuse via vacancy drag, although they show a lower temperature threshold than the late metals. This confirms that drag is the most common solute-vacancy coupling mechanism in iron at low temperatures, and this is likely to be confirmed as well for impurity diffusion in other transition metals.
\end{abstract}

DOI: 10.1103/PhysRevB.93.184302

\section{INTRODUCTION}

Ferritic and ferritic-martensitic (F/M) alloys are widely used in many industrial applications. They consist of bodycentered-cubic (bcc) iron alloys with varying concentrations of several impurities, most of which are purposely included in order to improve the alloy mechanical properties. Impurity diffusion plays a crucial role in determining such properties during fabrication, processing, and operation. Correlations with crystal defects can severely affect the diffusion process and hence the material macroscopic behavior. The effects in irradiated materials are even greater because of the strongly increased concentration of vacancies and self-interstitial atoms (SIA). For instance, the arising of solute drag by vacancies has been shown to be among the main causes for radiationenhanced or -induced solute precipitation [1-3], as well as for solute segregation at defect sinks [4-6], which in turn are responsible for hardening and embrittlement $[7,8]$. Solute-defect correlations can also substantially affect defect migration. As an example, the swelling-rate reduction in $\mathrm{FeCr}$ alloys with increasing $\mathrm{Cr}$ content has been ascribed to the strong correlation between $\mathrm{Cr}$ atoms and self-interstitial loops: diffusion of SIA's is slowed down by the correlation with solutes, and the recombination with vacancies is therefore enhanced [9]. A similar effect has been postulated for $\mathrm{Mn}$ impurities [10,11].

\footnotetext{
*messina@kth.se
}

Kinetic correlations between solutes and vacancies can lead to vacancy drag, i.e., to a coupled flux of solutes and vacancies in the same direction, as opposed to the inverseKirkendall diffusion mechanism [12]. Recent studies in dilute iron alloys have shown that vacancy drag can systematically arise in a wide range of conditions [13-15]. These studies are based upon the calculation of transport coefficients through the self-consistent mean-field (SCMF) method [16], which proved to be considerably more accurate than the traditional multifrequency models [17-19]. SCMF theory was applied for a systematic investigation of vacancy drag in bcc and facecentered-cubic (fcc) alloys [13,20]. In these works, the authors highlighted the predominant role of kinetic correlations over thermodynamic interactions in controlling and determining the arising of vacancy drag. This entails that the character of solute-vacancy interactions is not sufficient for an accurate prediction of flux coupling, but a full kinetic characterization of each single alloy is necessary. However, the conclusions therein are based on simple thermodynamic models, which are not necessarily representative of the behavior of "real" alloys. Such kind of kinetic studies can be performed with a higher accuracy if $a b$ initio methods are applied in the calculation of microscopic jump frequencies, on which the SCMF method relies.

Several examples of $a b$ initio-based studies of impurity diffusion in bec iron can be found in the literature [21-24]. However, such studies cover a limited set of impurities and do not treat flux-coupling phenomena. Flux coupling was more extensively investigated in [15,25], limitedly to 
some impurities that are of importance for the microstructure evolution of reactor pressure vessel (RPV) F/M steels. By means of a combined $a b$ initio SCMF approach, vacancy drag was found to systematically occur for most impurities $(\mathrm{Cu}$, $\mathrm{Mn}, \mathrm{Ni}, \mathrm{P}, \mathrm{Si}$ ) below a solute-dependent temperature threshold that is usually above $1000 \mathrm{~K}$ [15]. This could be directly linked to the observed formation of solute precipitates, even in the apparent absence of thermodynamic driving forces $[3,8]$.

Flux coupling is a complex kinetic phenomenon that depends on a large set of jump frequencies, for which clear physical trends have not been identified yet. The coupling strength and the threshold temperature could be correlated to first- and second-nearest-neighbor (nn) solute-vacancy interactions only in a qualitative way in [13]. However, the variation of the 2 nn solute-vacancy binding energy from one solute to another, as well as its effective impact on flux coupling, are not totally understood. The presence of a $2 \mathrm{nn}$ binding interaction in bcc crystals is usually regarded as a necessary condition for vacancy drag to take place [26,27]; however, it was shown in [13] that vacancy drag can occur even in the presence of some repulsive interactions, either at $1 \mathrm{nn}$ or $2 \mathrm{nn}$. The objective here is hence to improve the understanding of the binding energies, the jump frequencies, and flux coupling in dilute $\mathrm{Fe}(X)$ alloys by studying these properties at the electronic scale. Group-specific trends have already been evidenced for the cohesive energy, the bulk modulus, and the equilibrium atomic volume [28], for the solute-vacancy binding energies in Fe [26], for the solute-vacancy [27] and divacancy binding energies [29] in $\mathrm{W}$, and for solute diffusion in $\mathrm{Ni}[30,31]$. While the variation of many physical properties across the transition-metal series has a simple bell-shaped behavior with a maximum approximately in the middle of the series, $2 \mathrm{nn}$ binding energies have a linear behavior in contrast to the parabolic trends [26,29]. It is then interesting to investigate the effect of this anomalous trend on diffusion properties.

In addition, transport coefficients allow for an accurate first-principle-based calculation of low-temperature solute tracer diffusion coefficients. The latter are essential quantities for the modeling of radiation-response phenomena, but are usually not accessible experimentally below $750^{\circ} \mathrm{C}$. Recently, thanks to an advanced atom-probe technique [32], it was possible to improve the spatial resolution and accurately measure diffusion coefficients at temperatures stretching down to $550{ }^{\circ} \mathrm{C}$. However, many applications of irradiated materials require the knowledge of solute-diffusion coefficients at lower temperatures since the increased defect population accelerates diffusion phenomena that would otherwise be very slow. Given the lack of low-temperature data, diffusion coefficients are usually extrapolated from high-temperature measurements. In ferritic alloys, however, the extrapolation is normally based on a limited temperature range in the ferromagnetic (FM) region, and can therefore lead to errors of several orders of magnitude. Conversely, the transport coefficients calculated in this work, combined with a proper magnetic-transition model, allow for a reliable estimation of solute-diffusion coefficients for all the transition metals (TM), over a wide range of temperatures.

This work presents therefore an electronic study of vacancyassisted diffusion for all TM impurities in iron-based dilute alloys. Other solutes ( $\mathrm{Al}, \mathrm{Si}$, and $\mathrm{P}$ ) are also included in this analysis since they are often present in many types of $\mathrm{F} / \mathrm{M}$ steels, for a total of 26 impurities. The adopted dilute limit entails that impurity transport by vacancy clusters, as well as possible multisolute effects on the transport coefficients, are neglected. For each binary alloy, microscopic jump frequencies are computed with an $a b$ initio method. The trends of binding energies, migration barriers, and attempt frequencies as functions of the $d$-band filling are discussed. Afterwards, the transport coefficients are obtained with the SCMF method, and are used to determine the arising of vacancy drag and solute-segregation tendencies, as well as to provide diffusion coefficients in the low-temperature range. The several recurrent trends revealed by this systematic study allow for a deeper understanding of the physics of impurity diffusion in iron.

\section{METHODOLOGY}

\section{A. Mean-field model}

The kinetic analysis of this work is based on the calculation of transport coefficients $\left(L_{i j}\right)$. The latter describe the kinetic response of the system to a thermodynamic driving force, and can be expressed as

$$
J_{i}=-\sum_{j=1}^{N} \frac{L_{i j}}{k_{B} T} \nabla \mu_{j},
$$

where $J_{i}$ is the flux of species $i$ and $\nabla \mu_{j}$ is the chemical potential gradient of species $j$. The off-diagonal terms $L_{i j}$ $(i \neq j)$ describe the correlation between the fluxes of $i$ and $j$.

The transport coefficients (also called the Onsager coefficients) are obtained here in the framework of the SCMF theory [16]. In this theory, an alloy is described through a set of kinetic pair interactions, which embody a small perturbation from the thermodynamic equilibrium state. Such kinetic interactions are calculated by solving the corresponding kinetic equations in steady-state conditions. A detailed explanation of this method can be found in $[13,16]$, and its extension to nonhomogeneous driving forces is introduced in [33].

The key input parameters for the SCMF theory are the atomic jump frequencies in the local atomic environment (LAE) around the solute-vacancy pair. In analogy with the regular multifrequency models [17-19], the size of the LAE (and therefore the amount of jump types to be distinguished) is determined by a cutoff distance $R$, beyond which all thermodynamic interactions are neglected. Once $R$ is defined, the jumps to be distinguished are those occurring within or across the borders of the LAE. In [15], the cutoff radius was set to the $5 \mathrm{nn}$, and the results showed that, in those binary alloys, a cutoff to the $2 \mathrm{nn}$ is necessary and sufficient to provide an acceptable description of flux coupling and diffusion coefficients. 1nn models in bcc are not suitable because the $2 \mathrm{nn}$ site plays a relevant role in vacancy-solute coupled diffusion, whereas the cutoff extension to the $5 \mathrm{nn}$ only slightly improved the results, to the cost of a considerable increase of the computational time. For this reason, the cutoff radius is set in this work to the $2 \mathrm{nn}$, leading to the typical nine-frequency configuration depicted in Fig. 1. It is worth pointing out that in precedent 2 nn multifrequency models the kinetic interactions are not fully accounted for, whereas the 


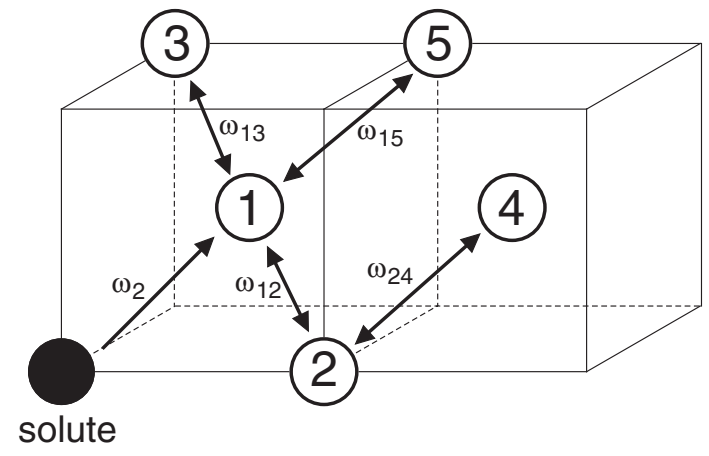

FIG. 1. Nine-frequency model enforced in this work for the calculation of the transport coefficients, in a $2 \mathrm{nn}$ thermodynamic model. The numbers in the circles mark the possible nearest-neighbor positions of the vacancy before the jump. The background iron jump frequency $\left(\omega_{0}\right)$ outside the solute LAE is not shown.

application of SCMF theory yields the correct flux-coupling tendencies, as was shown in [15].

Each migration is modeled as a thermally activated event with frequency:

$$
\omega_{i j}=v_{\mathrm{x}} \exp \left(-\frac{E_{i j}^{\mathrm{mig}}}{k_{B} T}\right) .
$$

The migration energies $E_{i j}^{\mathrm{mig}}$ and attempt frequencies $v_{\mathrm{Fe}}, v_{\mathrm{x}}$ are obtained by means of density functional theory (DFT) $a b$ initio calculations. Given the high computational cost required by attempt frequency DFT calculations, the attempt frequencies for iron jumps in the vicinity of a solute atom $\left(\omega_{i j} \neq \omega_{0}, \omega_{2}\right)$ are assumed to be equal to $\nu_{\mathrm{Fe}}$.

The SCMF method requires the transition rates to be calculated in conditions of thermodynamic equilibrium, and in such conditions each elementary transition must be balanced by its reverse process (detailed-balance principle). A manipulation of the DFT migration barriers is therefore necessary. It is achieved by adding the DFT-computed binding energy $E_{z}^{\mathrm{b}}$ to each migration barrier of type $E_{z y}^{\mathrm{mig}}$, with $z>R$ and $y \leqslant R$, so to set $E_{z}^{\mathrm{b}}$ to zero. For instance, since DFT yields $E_{3}^{\mathrm{b}}(\mathrm{Mo})=-0.02 \mathrm{eV}$ (attractive), the migration energy of the jump $\omega_{31}$ is lowered by $0.02 \mathrm{eV}$ in order to remove this residual interaction at $3 \mathrm{nn}$. Clearly, this procedure has little effect on the results as long as the residual interactions beyond $R$ are small [15].

Once the set of jump frequencies is obtained, the transport coefficients are computed in the SCMF framework with the $3 \mathrm{nn}-3 \mathrm{nn}$ kinetic model, which includes the kinetic interactions among atoms within the $3 \mathrm{nn}$ of $3 \mathrm{nn}$ sites (with respect to the vacancy initial and final position). The matrix formulation of this model is to be found in the Appendix of [13]. The vacancy-drag coefficient is defined as $L_{x \mathrm{v}} / L_{x x}$, with $L_{x \mathrm{v}}=$ $-\left(L_{\mathrm{Fe}, x}+L_{x x}\right)$. This coefficient is positive under vacancydrag conditions, and negative otherwise.

The transport coefficient matrix allows also for the prediction of radiation-induced segregation (RIS) profiles. In thermal-equilibrium conditions, solutes might segregate on defect sinks (e.g., dislocations or grain boundaries) in case of an attractive thermodynamic interaction with the sink. This phenomenon is referred to as equilibrium segregation. Under irradiation, the net flux of defects towards sinks is nonzero, hence, the kinetic coupling with impurities can lead to an additional enrichment or depletion tendency, superposed on the equilibrium segregation profile (as for example in [34]). Here, the RIS due to solute coupling with vacancies is analyzed in terms of partial diffusion coefficient (PDC) ratio [35], which can be written in the dilute limit as

$$
\frac{d_{x \mathrm{v}}}{d_{\mathrm{Fe}, \mathrm{v}}}=\frac{l_{\mathrm{Fe}, x}+l_{x x}}{L_{\mathrm{Fe}, \mathrm{Fe}}^{0}},
$$

where $l_{\mathrm{Fe}, x}=L_{\mathrm{Fe}, x} / c_{x}$ and $l_{x x}=L_{x x} / c_{x} . L_{\mathrm{Fe}, \mathrm{Fe}}^{0}$ is the uncorrelated, solute-independent part of the $L_{\mathrm{Fe}, \mathrm{Fe}}$ coefficient, and $c_{x}$ is the atomic solute concentration. The PDC ratio enters the general RIS balance equation [15]

$$
\frac{\nabla c_{x}}{\nabla c_{\mathrm{v}}}=\frac{c_{\mathrm{Fe}} c_{x} d_{\mathrm{Fe}, v} d_{\mathrm{Fe}, i}}{\left(c_{\mathrm{Fe}} d_{\mathrm{Fe}, i} D_{x}+c_{x} d_{x i} D_{\mathrm{Fe}}\right)}\left(\frac{d_{x v}}{d_{\mathrm{Fe}, v}}-\frac{d_{x i}}{d_{\mathrm{Fe}, i}}\right),
$$

where the indices $v$ and $i$ stand for vacancies and interstitials, respectively. Equation (4) is valid for ideal sinks, i.e., sinks with infinite defect absorption capacity and no bias. The full derivation and the definition of the interdiffusion coefficients $D_{\mathrm{Fe}}, D_{x}$ can be found in $[15,35]$. Here, the amplitude prefactor is neglected and the PDC ratio for interstitials is set to 1 . This is equivalent to neglecting the enrichment or depletion tendency induced by interstitial transport, which might be significant for solutes that form stable dumbbells such as $\mathrm{P}, \mathrm{Mn}$, and Cr $[21,26]$.

The solute tracer diffusion coefficient is calculated as

$$
D_{x}^{*}=\frac{L_{x x}}{n c_{x}},
$$

where $n$ is the iron atomic density. In the dilute limit, $L_{x x}$ (as well as $L_{\mathrm{Fe}, x}$ ) is directly proportional to the probability of forming a vacancy-solute pair, which is in turn proportional to $c_{x}$ and $c_{\mathrm{v}}$. $D_{x}^{*}$ is therefore independent of $c_{x}$. It can be equivalently expressed as [36]

$$
D_{x}^{*}=a_{0}^{2} c_{\mathrm{v}} f_{x} \omega_{2} \exp \left(-\frac{E_{1 \mathrm{nn}}^{\mathrm{b}}}{k_{B} T}\right),
$$

where $c_{\mathrm{v}}$ is the vacancy concentration, $f_{x}$ the solute-diffusion correlation factor, and $E_{1 \mathrm{nn}}^{\mathrm{b}}$ the $1 \mathrm{nn}$ solute-vacancy interaction energy (taken as positive when repulsive and negative otherwise). The correlation factor takes into account the probability that atomic jumps do not occur randomly in each direction because of geometric reasons as well as solutevacancy correlations. Therefore, the whole effect of kinetic correlations on solute diffusion is included in this factor. The correlation factor $f_{x}$ yielded by SCMF theory is provided in the Appendix as a function of the jump frequencies. Contrary to the commonly used Le Claire's formula [17], this expression does not assume that $\omega_{42}=\omega_{0}$, thus allowing for a more accurate calculation of $f_{x}$. The tracer self-diffusion coefficient can be obtained with the same methodology, by introducing a "mock" solute atom with null binding energy and jump frequency $\omega_{0}$.

The vacancy concentration $c_{\mathrm{v}}$ is here taken to be that of nonirradiation thermodynamic-equilibrium conditions

$$
c_{\mathrm{v}}=c_{\mathrm{v}}^{\mathrm{eq}}=\exp \left(-\frac{E_{\mathrm{v}}^{\mathrm{f}}}{k_{B} T}\right) \exp \left(\frac{S_{\mathrm{v}}^{\mathrm{f}}}{k_{B}}\right),
$$




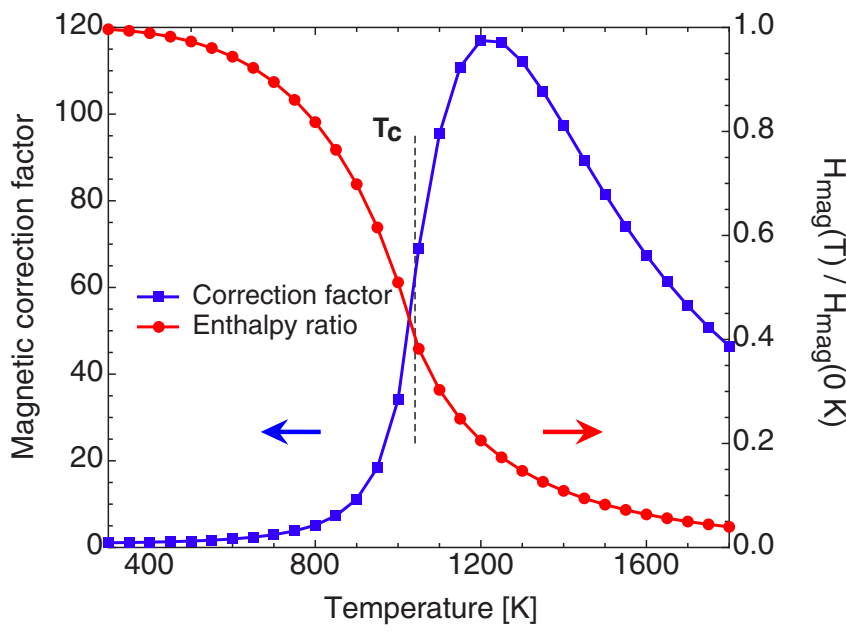

FIG. 2. (Blue squares) Correcting factor $\exp [\alpha H(T)]$ to selfdiffusion and solute-diffusion coefficients due to the finitetemperature magnetic transition. (Red circles) Relative excess magnetic enthalpy obtained with the Hillert-Jarl model [38]. $T_{\mathrm{C}}=$ $1043 \mathrm{~K}$ marks the Curie temperature in iron.

for the sake of consistency with diffusion experiments. The formation enthalpy and entropy $E_{\mathrm{v}}^{\mathrm{f}}$ and $S_{\mathrm{v}}^{\mathrm{f}}$ are obtained as well via DFT calculations. The variation of $E_{\mathrm{v}}^{\mathrm{f}}$ due to the solute concentration can be neglected in the dilute-limit calculation of vacancy-solute flux coupling. In irradiated materials, the vacancy concentration is fixed by the external irradiation and the dynamic balance with other defects. At any rate, since each transport coefficient is proportional to $c_{\mathrm{V}}$, the flux-coupling tendencies here obtained are not affected by an increased vacancy population.

As to account for the effects of ferromagnetic to paramagnetic (PM) transition in iron, a finite-temperature model inspired by the work of Sandberg et al. [37] is here adopted. The total vacancy diffusion activation energy in pure iron $Q_{0}^{\mathrm{F}}=E_{\mathrm{v}}^{\mathrm{f}}+E_{\mathrm{v}}^{\mathrm{m}}$ decreases proportionally to the magnetic excess enthalpy $H^{\mathrm{mag}}$ :

$$
Q(T)=Q_{0}^{\mathrm{F}}-\alpha H(T),
$$

with $\alpha=Q_{0}^{\mathrm{F}}-Q^{\mathrm{P}}$ and $H(T)=1-H^{\mathrm{mag}}(T) / H^{\mathrm{mag}}(0 \mathrm{~K})$. $Q^{\mathrm{P}}$ is the vacancy diffusion activation energy in a fully PM state $\left(Q^{\mathrm{P}}=2.26 \mathrm{eV}\right.$ according to recent $a b$ initio calculations [37]). The magnetic excess enthalpy is calculated by means of the Hillert-Jarl model [38], reported in the Appendix. Equation (8) was developed for self-diffusion in iron, but can be also applied to solute diffusion, under the assumption that one solute atom has a negligible effect on the correlation between the magnetic state and vacancy migration properties. The minor electronic contribution to the activation energy treated in [37] is here neglected. The vacancy mobility, and hence the self-diffusion and solute-diffusion coefficients, are increased by the temperature-dependent factor $\exp [\alpha H(T)]$ shown in Fig. 2. The same figure reports as well the value of the factor $H(T)$. The discontinuity at the Curie temperature is due to the two different Hillert-Jarl expressions for the magnetic excess enthalpy used in the FM and PM regions. Short-range ordering is represented by the residual magnetic enthalpy above the Curie temperature.

\section{B. $A b$ initio method}

The DFT calculations are performed with the Vienna $a b$ initio simulation package (VASP) [39-41] on a plane-wave basis, by making use of projector augmented wave (PAW) pseudopotentials $[42,43]$. The exchange-correlation function is modeled with the Perdew-Burke-Ernzerhof (PBE) parametrization [44] of the generalized gradient approximation. The plane-wave cutoff is set to $300 \mathrm{eV}$ and the Brillouin zone is sampled with a $3 \times 3 \times 3 k$-point mesh. The calculations are spin polarized and are performed in a 127-atom supercell (unless otherwise specified), allowing for atomic relaxations but restraining the cell shape and volume. Standard potentials from the VASP library are employed for all the elements included in this study. The migration barriers are calculated with the nudged-elastic band (NEB) method [45,46] and the climbing image algorithm [47], using three intermediate images. In this way, the migration barriers can be determined to an accuracy of $5 \mathrm{meV}$ or less. More details can be found in [15].

The vacancy formation and migration energies in pure iron were calculated in [15] and are here unchanged. The solute-vacancy binding energy for a given $i$-nn configuration is obtained as

$$
E_{i \mathrm{nn}}^{b}=E_{\mathrm{v}+x(i \mathrm{nn})}^{N-2}-E_{\mathrm{v}}^{N-1}-E_{x}^{N-1}+E^{N},
$$

where the terms on the right-hand side refer to the supercell energy with a vacancy and a solute atom, one vacancy, one solute atom, and with no defects nor solutes, respectively.

The vacancy formation entropy and the attempt frequencies for iron and solute migration are determined by means of DFT frozen-phonon calculations. In the harmonic approximation to transition-state theory (TST), the vacancy formation entropy in pure iron is obtained from the vibrational frequencies of the defected (I) and undefected (U) supercell [48]

$$
\frac{S_{\mathrm{v}}^{\mathrm{f}}}{k_{B}}=-\left[\ln \left(\prod_{k=1}^{3 N-3} v^{\mathrm{I}}\right)-\frac{N}{N+1} \ln \left(\prod_{k=1}^{3(N+1)-3} v^{\mathrm{U}}\right)\right],
$$

and the attempt frequency of a given migration event is derived from the vibrational frequencies of the initial (I) and saddlepoint (S) configurations [49]

$$
v_{x}=\frac{\prod_{k=1}^{3 N-3} v_{k}^{\mathrm{I}}}{\prod_{k=1}^{3 N-4} v_{k}^{\mathrm{S}}} .
$$

$N$ is the number of atoms (127 in a $4 \times 4 \times 4$ supercell), and the index $k$ scans through the degrees of freedom of the system. The vibrational frequencies are computed in the quasiharmonic approximation by diagonalizing the Hessian matrix. Each element $k_{i j}$ of this matrix is given by the derivative of the force on atom $i$ with respect to a small displacement of atom $j$ in one of the three spatial directions:

$$
k_{i j}=\frac{1}{\sqrt{m_{i} m_{j}}} \frac{\partial F_{i}}{\partial r_{j}} .
$$

Four displacements of \pm 0.015 and $\pm 0.030 \AA$ are applied on each atom in each direction, and the force is interpolated with the least-square method. Given the extreme sensibility with respect to the force convergence criterion, the initial and undefected configurations are relaxed so that the force on 
each atom is lower than $0.001 \mathrm{eV} / \AA$. This value is one order of magnitude higher than in similar calculations performed by Lucas et al. [50]. Tests on 15-atom cells with varying cutoff and $k$-point mesh size confirmed that the vibrational properties are quite well converged at $0.001 \mathrm{eV} / \AA$. With this fixed force-convergence criterion, the phonon calculations in pure iron are run in simulation cells of increasing size, in order to check the box-size effect. For this purpose, simulation cells of $15,53,127$, and 249 atoms are used, and the $k$-point mesh is adjusted accordingly $(7 \times 7 \times 7$ for 15 atoms, $5 \times 5 \times 5$ for 53 atoms, and $3 \times 3 \times 3$ for 127 and 249 atoms), with a fixed cutoff energy of $350 \mathrm{eV}$. Because of the large computational cost, the solute attempt frequencies are calculated in a 53-atom supercell only. At the moment, the same computation-time limitation prevents the possibility of calculating the attempt frequency of pure $\mathrm{Fe}$ in a 249-atom cell.

\section{RESULTS AND DISCUSSION}

\section{A. Bulk-iron properties}

The bulk properties of iron obtained or adopted in this work are listed in Table I. The lattice parameter and the vacancy activation energy in FM state were already shown to be in agreement with experiments and previous DFT calculations [15]. The PM activation energy was DFT calculated in [37], as an average over a set of several randomly assigned spin configurations, so to simulate the PM state.

The phonon-related quantities $\left(S_{\mathrm{v}}^{\mathrm{f}}\right.$ and $\left.\nu_{\mathrm{Fe}}\right)$ are calculated in simulation cells of increasing size, and in both cases they reach an acceptable convergence. Based on these values, it is possible to assess the accuracy of the calculations to less than $\pm 0.2 k_{B}$ and $\pm 1 \mathrm{THz}$, respectively. The obtained vacancy formation entropy of $4.6 k_{B}$ is larger than early calculations using semiempirical potentials (SEP) and ranging between 1.5 and $2.6 k_{B}$ [51-53]. It is, however, in better agreement with previous DFT calculations $\left(S_{\mathrm{v}}^{\mathrm{f}}=4.08 k_{B}\right)$ [50], confirming the qualitative difference between DFT and SEP. In addition, this value is also compatible with the estimation provided in [54] based on a TST-based analysis of self-diffusion data. According to this analysis, the sum of the vacancy formation and migration entropy should be lower than $5 k_{B}$. As for the attempt frequency, the calculated value is in very good agreement with recent DFT calculations $(12 \mathrm{THz})$ [55].

\section{B. Solute-vacancy interactions}

The calculated solute-vacancy interaction energies are shown in Fig. 3. They are in good agreement with previous calculations $[23,26,56,57]$ performed with various first-principles methods, and with the experimental values listed in $[15,26]$. With the exception of the $2 \mathrm{nn}$, the interaction is always attractive, reaching particularly strong values at the two ends of the band. Three types of $1 \mathrm{nn}-2 \mathrm{nn}$ solute-vacancy interactions can be therefore distinguished: binding-repulsive (early TM's), binding-binding (late TM's), and weak interactions (the metals in-between, except Mn). To the second category belong also $\mathrm{P}$ and $\mathrm{Si}$, whereas $\mathrm{Al}$ can be included into the first class. According to the traditional assumptions, the early TM's should not be dragged by vacancies because of the $2 \mathrm{nn}$ repulsion, in spite of the (in some cases) very strong $1 \mathrm{nn}$ binding interaction. The diffusion behavior of the early TM's is therefore suitable for confirming or disproving this theory.

$\mathrm{Co}$ and $\mathrm{Ni}$ are anomalous as they are characterized by a higher binding energy at $2 \mathrm{nn}$ than $1 \mathrm{nn}$. Such anomalies, repeatedly found in past works [26,57], can now be explained thanks to the peculiar shape of the 2 nn group trend, i.e., a somewhat linear descent from repulsion to attraction. Similar calculations of solute-vacancy interactions in bcc tungsten also showed an anomalous $2 \mathrm{nn} d$-band trend, as well as a separation in behavior between early and late TM's [27].

On the other hand, the group trends of the other nn interactions follow a parabolic curve for $4 d$ and $5 d$ metals, and an M-shaped curve for the $3 d$ series. Such shapes are observed in many TM properties such as cohesive energy [58], bulk modulus [58], solute size factor in Fe [26], or solute migration energy in $\mathrm{Ni}$ [30], and will reappear as well in the diffusion properties presented in the later sections of this paper. The parabolic trend was shown to be associated with the second moment of the density of states (DOS), in the framework of a tight-binding model [28,59]. Conversely, the behavior of the $3 d$ metals in Fe is strongly affected by magnetic interactions, which are absent in the $4 d$ and $5 d$ series [26]. In particular, the $\mathrm{M}$ shape is determined by the atypical behavior of $\mathrm{Mn}$. This was shown to be also the case for interactions in a $\mathrm{Ni}$ matrix [30]. While $\mathrm{Ti}$ and $\mathrm{V}$ show an analogous behavior to the early $4 d$ and $5 d$ TM solutes, and $\mathrm{Cu}$ that of a late TM solute, one can note that the $3 d$ solutes with significant magnetic coupling diverge from the $4 d$ and $5 d$ trends. Namely,

TABLE I. Bulk properties in pure iron, computed or adopted in this work.

\begin{tabular}{|c|c|c|c|c|}
\hline Quantity & Value & & & \\
\hline Lattice parameter $a_{0}$ & $2.831 \AA^{\mathrm{a}}$ & & & \\
\hline Vacancy formation enthalpy $E_{\mathrm{v}}^{\mathrm{f}}$ & $2.18 \mathrm{eV}^{\mathrm{a}}$ & & & \\
\hline Vacancy migration energy $E_{\mathrm{v}}^{\mathrm{mig}}$ & $0.70 \mathrm{eV}^{\mathrm{a}}$ & & & \\
\hline Ferromagnetic activation energy $Q_{\mathrm{v}}^{\mathrm{F}}$ & $2.88 \mathrm{eV}^{\mathrm{a}}$ & & & \\
\hline Paramagnetic activation energy $Q_{\mathrm{v}}^{\mathrm{F}}$ & $2.26 \mathrm{eV}^{\mathrm{b}}$ & & & \\
\hline Entropic quantities (this work) & 15 at. & 53 at. & 127 at. & 249 at. \\
\hline Vacancy formation entropy $S_{\mathrm{v}}^{\mathrm{f}}\left(k_{\mathrm{B}}\right)$ & 4.90 & 4.54 & 4.83 & 4.62 \\
\hline Attempt frequency $v_{\mathrm{Fe}}(\mathrm{THz})$ & 4.19 & 10.8 & 11.6 & \\
\hline Vacancy diffusion prefactor $D_{\mathrm{Fe}}^{0}\left(\mathrm{~cm}^{2} / \mathrm{s}\right)$ & 0.45 & 0.81 & 1.16 & \\
\hline
\end{tabular}

${ }^{\mathrm{a}}$ Reference [15].

${ }^{b}$ Reference [37]. 

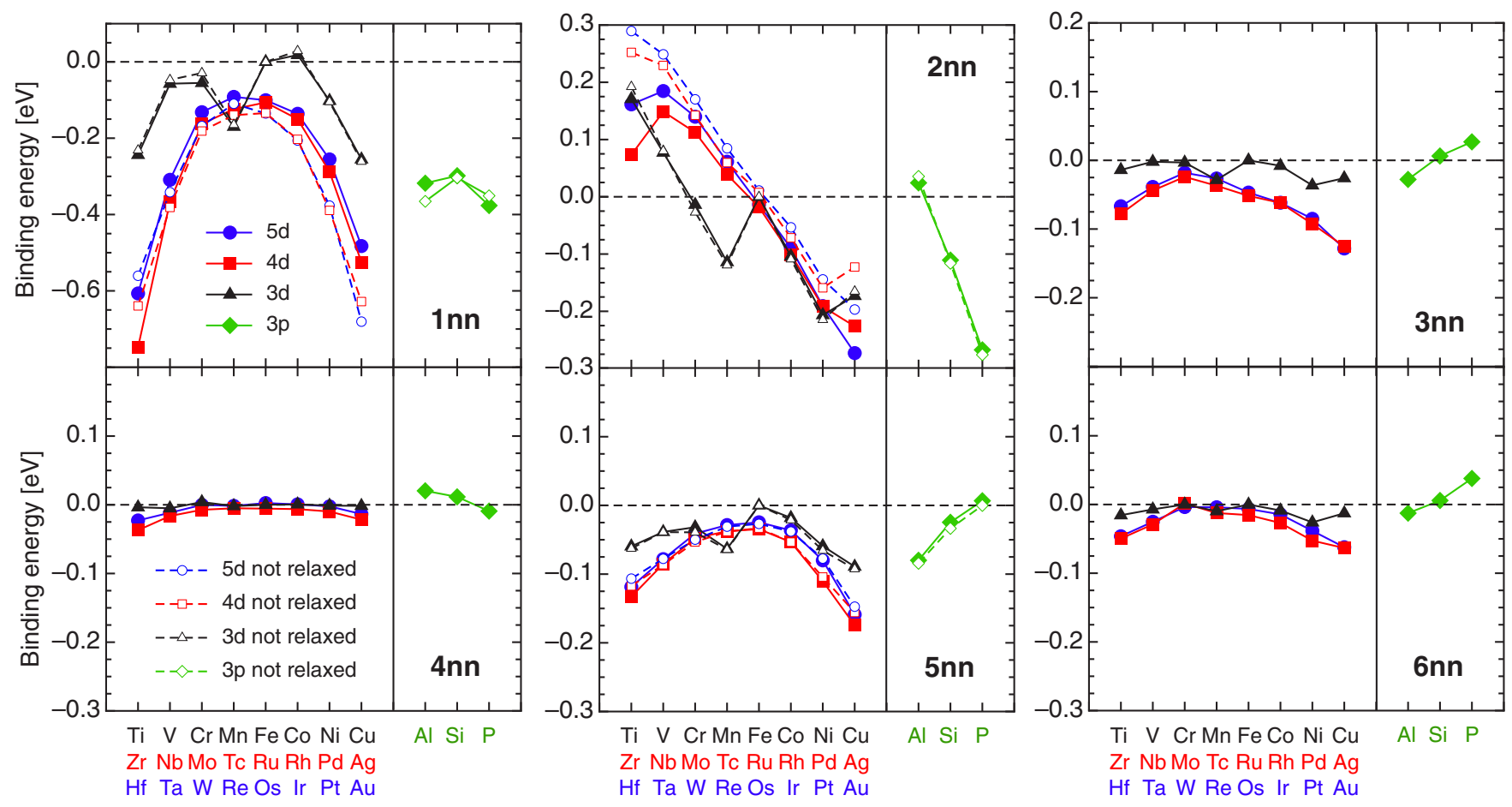

FIG. 3. DFT-calculated solute-vacancy interaction energies in iron (full symbols), and computed by neglecting the relaxation of the cell due to the solute presence (open symbols). Negative values stand for attractive interactions.

the antiferromagnetic solutes $(\mathrm{Cr}, \mathrm{Mn})$ have a purely binding behavior, to an extent that clearly depends on the magnetic moment. On the other hand, the FM solutes (Co, Ni) are strongly bound at $2 \mathrm{nn}$ only, and their behavior tends to the late TM's trend as the magnetic moment decreases.

Solute-defect interactions are usually explained according to strain-relief arguments: oversized atoms are expected to bind in compressed positions ( $1 \mathrm{nn}, 3 \mathrm{nn}, 5 \mathrm{nn})$ and repulse in tensile ones ( $2 \mathrm{nn}, 4 \mathrm{nn})$. However, the $2 \mathrm{nn}$ interaction of the late metals contradicts this rule. According to [26], this has an electronic origin: the late elements perturb the charge distribution around the vacancy in a different fashion with respect to the early elements.

In order to rule out the size-strain effects, the electronic contribution to the total binding energy is also shown in Fig. 3 for $1 \mathrm{nn}, 2 \mathrm{nn}$, and $5 \mathrm{nn}$ (empty markers). This contribution is obtained by calculating the binding energy without allowing for atomic relaxations, in order to evidence the solute-size effect. As can be seen in the figure, the electronic contribution is clearly the dominant factor, as has already been shown for some of the impurities [56]. The difference between the electronic contribution and the total interaction increases with solute size, again in agreement with [56]. On the other hand, the size effect seems to be negligible for the $3 d$ metals, which are fairly close matched in size with Fe. The interaction is nearly independent of solute size also for the $p$ elements, as was already mentioned for $\mathrm{P}$ [21].

The effect of ionic relaxations cannot be rationalized with simple size arguments. For instance, the 1 nn interaction becomes stronger for $\mathrm{Zr}$ and $\mathrm{Hf}$, and increasingly weaker for the other elements. This is surely due to additional effects of the electronic rearrangement after relaxation, and might also be affected by the larger substitution energy of the late
TM's [26]. Moreover, the linear trend of the $2 \mathrm{nn}$ interaction has a clear electronic origin, and the total energy is systematically more binding than the electronic contribution, in spite of the tensile character of the $2 \mathrm{nn}$ strain field [26]. Following the tight-binding model of [28], the linear shape of the $2 n n$ interaction is caused by the contribution of higher-than-second moments of the DOS, which in turn can be related to a strong angular character of the electronic interactions. Such character might also explain the deviation from the trend of the large-sized elements ( $\mathrm{Zr}$, Hf on one side and Ag, Au on the other side). It is worth mentioning that some anomalies in the properties of Ag and Au have already been observed [26].

Figure 3 shows as well a residual interaction at $3 \mathrm{nn}$ and especially at $5 \mathrm{nn}$. In the latter case, the iron atom between the solute and the vacancy might be playing an important role. This is in disagreement with previous DFT-PW91 calculations [26], which yielded negligible $3 \mathrm{nn}$ and $5 \mathrm{nn}$ interactions. However, additional testing of such potentials confirms this residual interaction. The box-size effect was found to be negligible (no more than $0.02 \mathrm{eV}$ ) by repeating the binding-energy calculations in a 249-atom supercell for some selected large solutes. This proves that indeed large-sized solutes are characterized by a long-ranged interaction with vacancies, which is dominated by electronic effects of the second-moment type. This finding suggests that, for such solutes, a 2 nn diffusion model might not suffice to fully describe the solute-vacancy kinetic interactions.

\section{Migration barriers}

The solute migration barriers (for jump $\omega_{2}$ ) as functions of the $d$-band filling are depicted in Fig. 4, alongside the corresponding attempt frequencies. The group trends have very similar shapes to those of the $1 \mathrm{nn}$ binding energy: a parabolic 

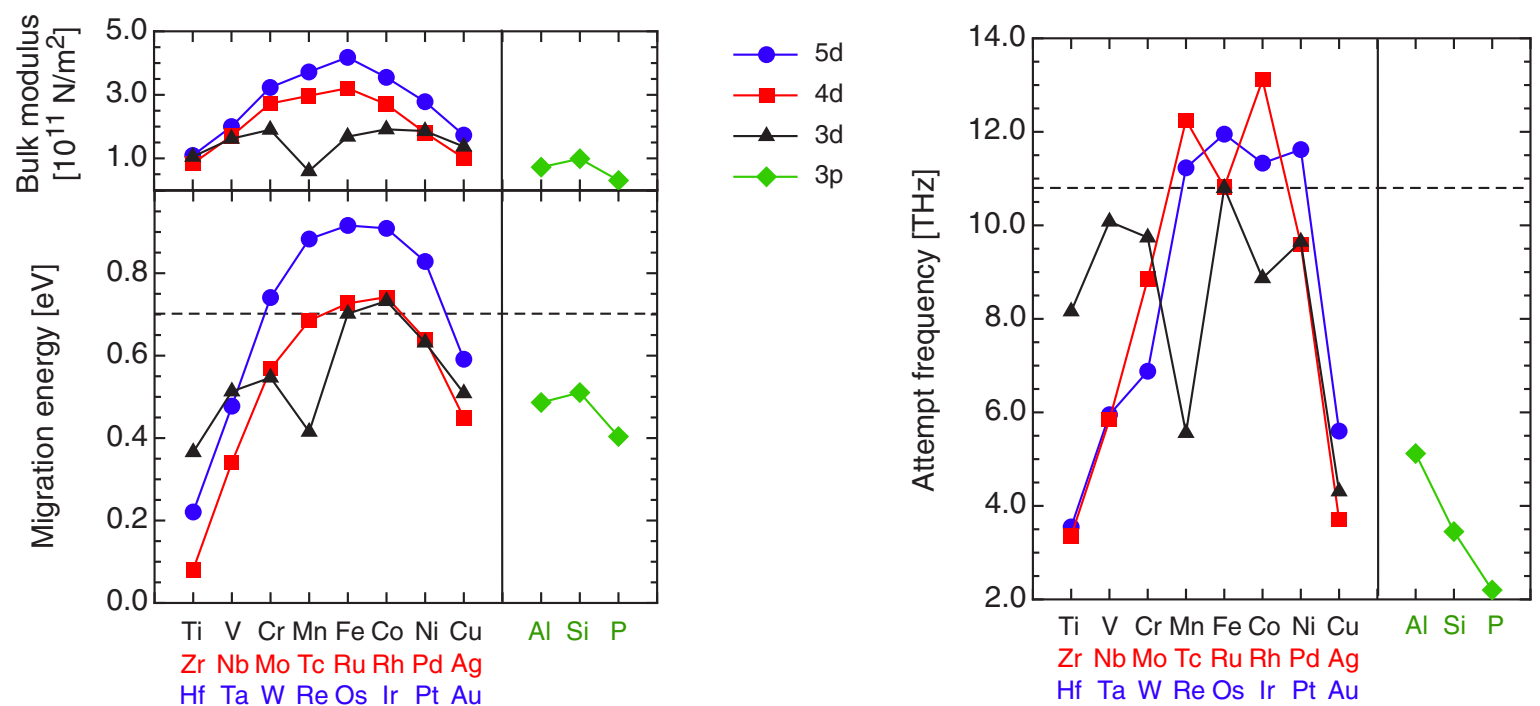

FIG. 4. DFT-computed solute migration barriers and attempt frequencies $\left(\omega_{2}\right)$. In the above-left panel, experimental bulk moduli of the pure elements [58]. The dashed lines represent the values in pure Fe.

function for the $4 d$ and $5 d$ elements, and an M-shaped curve for the $3 d$ ones. These shapes suggest that large solutes diffuse faster, as their migration barrier is lower. On the other hand, also the small solutes ( $\mathrm{P}$ and $\mathrm{Si}$ ) have a lower barrier. This general trend is very similar to that of solute migration barriers in a nickel matrix [30]. Although the effect is partially compensated by the opposite trend in the attempt frequencies, it can be also observed in the experimental diffusion data of solutes in Fe (see Fig. 12).

That large atoms are faster is surely counterintuitive, as one would expect smaller atoms to induce less strain in the crystal and have therefore an easier way while migrating. Janotti et al. [30] explained this phenomenon in terms of solute compressibility. They demonstrated that the saddle-point energy is not much affected by the misfit strain, but is instead dominated by how much the solute can be compressed at the saddle-point position. This was also the case for solute migration in $\mathrm{Al}$ [60], and holds here as well in an iron matrix, because of the electronic origin of this effect. Band-center solutes are less compressible because of the presence of directional bonds, which are not there for the band-end solutes. For comparison, the experimental bulk moduli of each element (in the pure material) are reported in the above-left panel of Fig. 4. It is clear that the shapes of the migration barrier trends for all the TM series are correlated with the solute bulk moduli, or their compressibilities. The lower compressibility of the $5 d$ row with respect to the $4 d$ row is the cause for the systematically higher migration barriers of $5 d$ metals, in spite of the similar chemical properties, and the difference increases towards the center. From a tight-binding standpoint, the parabolic behavior of the bulk modulus, and as a consequence of the migration barrier, is determined by the DOS second moment. As was the case for the Ni matrix [30], the saddle-point distance between the migrating solute and the $1 \mathrm{nn}$ iron atoms is only weakly dependent on the solute type, which confirms the low influence of the size factor. The asymmetry of the curve, which was observed as well in $\mathrm{Ni}$, can be attributed to the higher substitution energy [26] and to the lower cohesive energy [58] of the late TM's. Certainly, the size factor that is commonly proposed as argument to explain interactions and migration barriers provides a negligible contribution. For what concerns the $3 d$ metals, the crossing with the $4 d$ and $5 d$ curves is observed for both the bulk modulus and $E_{2}^{\text {mig }}$, but their behavior cannot be explained by compressibility only. It is most likely affected by complex magnetic interactions. For the same reasons as the binding energy case, the peculiar electronic interactions of $\mathrm{Mn}$ determine the local minimum and the $\mathrm{M}$ shape of the $3 d$ trend.

A few elements are characterized by a higher migration barrier than $\mathrm{Fe}$, namely, $\mathrm{Co}, \mathrm{Ru}, \mathrm{Rh}$, and all $5 d$ metals from $\mathrm{W}$ to Pt. However, as will be discussed in the next sections, this does not entail necessarily a slower diffusion than selfdiffusion. On the other hand, a few early TM's, especially $\mathrm{Zr}$, have very small migration barriers $(0.08 \mathrm{eV})$. The behavior of this solute tends to the limiting case of yttrium, whose binding with a vacancy is so strong that it is not located in a substitutional position, rather it relaxes towards the middle of two vacant spaces. The "migration barrier" of $Y$ is so low (below $0.01 \mathrm{eV}$ ) that the diffusion mechanism is no more an exchange with a monovacancy, but rather a migration of the whole Y-vacancy complex [61]. The Zr-vacancy configuration is very stable since $\mathrm{Zr}$ relaxes quite far into the middle of the vacant space $(0.4 \AA$, or $16 \%$ of the $1 \mathrm{nn}$ distance), and the probability of vacancy escape is negligible (the escape barriers for $\omega_{12}, \omega_{13}$, and $\omega_{15}$ are much higher than $\omega_{2}$, and the reassociation barriers $\omega_{31}$ and $\omega_{51}$ are much lower than $\left.\omega_{0}\right)$. This suggests that the vacancy is actually trapped next to a $\mathrm{Zr}$ solute up to high temperatures, and diffusion could take place with a mechanism similar to that of $\mathrm{Y}$, in which case the diffusion model here applied would be inaccurate.

The iron migration barriers in the vicinity of a solute atom are shown in Fig. 5, according to the jump-frequency nomenclature of Fig. 1. For these jumps, the iron attempt frequency is assumed to be independent from the LAE of the migrating atom. In general, it is possible to observe a very strong association tendency (low migration barriers for the 

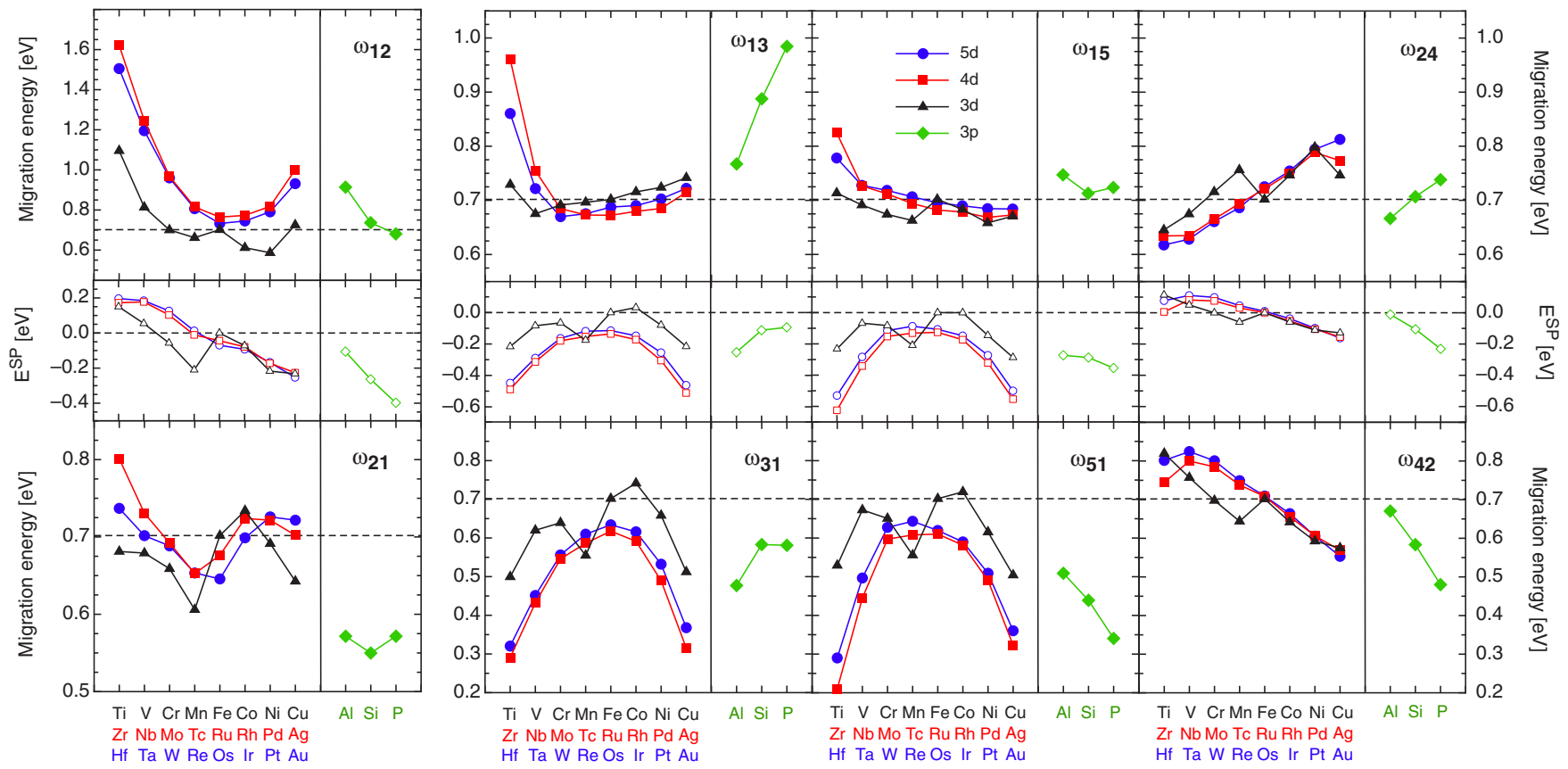

FIG. 5. DFT-computed iron migration barriers $\omega_{i j}$ in the vicinity of a solute atom, according to the jump-frequency nomenclature of Fig. 1 . The dashed lines mark the values in pure $\mathrm{Fe}\left(v_{\mathrm{Fe}}\right)$. In the middle panels, the saddle-point contribution to each migration barrier, obtained by removing the initial-state binding energy and the pure-Fe vacancy migration barrier $\left(E_{i j}^{\mathrm{SP}}=E_{j i}^{\mathrm{SP}}\right)$.

reverse jumps $\omega_{31}$ and $\left.\omega_{51}\right)$ and also a strong resistance against dissociation for the early TM's (high barriers for the $\omega_{12}$ and $\omega_{13}$ jumps). Given also the low solute migration barriers, very strong solute-vacancy correlations are expected for the early TM's. For Co, the lack of $1 \mathrm{nn}$ interaction and the combination of the iron jump frequencies show that the Co-vacancy $2 \mathrm{nn}$ configuration can represent a trapping configuration, which makes Co diffusion in iron considerably more difficult.

The group trends can be explained by separating the saddlepoint energy properties from the binding energy influence. For this purpose, the saddle-point (SP) contribution to the total migration barrier can be isolated by removing the initial-state binding energy in the following way:

$$
E_{i j}^{\mathrm{SP}}=E_{i j}^{\mathrm{mig}}-E_{0}^{\mathrm{mig}}-E_{i}^{\mathrm{b}},
$$

where $E_{0}^{\mathrm{mig}}=0.70 \mathrm{eV}$ is the migration energy in pure iron. $E_{i j}^{\mathrm{SP}}$ embodies therefore the deviation from the saddle-point energy in pure $\mathrm{Fe}$, due to the interactions of the solute with the hopping vacancy. Moreover, since $E_{i j}^{\mathrm{mig}}-E_{j i}^{\mathrm{mig}}=E_{j}^{\mathrm{b}}-E_{i}^{\mathrm{b}}$ due to the detailed-balance principle, it is easy to derive that $E_{i j}^{\mathrm{SP}}=E_{j i}^{\mathrm{SP}}$.

$E_{i j}^{\mathrm{SP}}$ is shown for each jump type in the middle panels of Fig. 5. The trends show a smooth variation with the $d$-band filling that closely resembles that of the binding energies. Namely, $E_{13}^{\mathrm{SP}}$ and $E_{15}^{\mathrm{SP}}$ are very similar to the $1 \mathrm{nn}$ binding energy, whereas $E_{12}^{\mathrm{SP}}$ and $E_{24}^{\mathrm{SP}}$ are remarkably close to $E_{2 \mathrm{nn}}^{\mathrm{b}}$. Even the magnitude of $E_{12}^{\mathrm{SP}}$ matches that of $E_{2 \mathrm{nn}}^{\mathrm{b}}$, while the magnitudes of the other jumps are dampened. This suggests that the saddle-point electronic interactions are similar to those occurring in the corresponding end states, and that the $2 \mathrm{nn}$ interaction dominates for the transitions to and from the $2 \mathrm{nn}$ configuration. Such interactions progressively fade out for saddle points that are further away from the solute.
The final shapes of the migration energy trends are then determined by the combination of the SP trends and the binding energy ones. For the $\omega_{13}$ and $\omega_{15}$ jumps, the 1 nn binding energy contribution is opposed, leading to values close to the background $\omega_{0}$, with the exception of the early TM's for which the $1 \mathrm{nn}$ binding energy is very strongly attractive. The situation is more complex for the $\omega_{12}$ and $\omega_{21}$ jumps. For the former, the superposition of SP and 1nn binding energy yields smooth trends with very high barriers, whereas for the latter the $2 \mathrm{nn}$ binding energy contribution has opposite sign and causes a very sharp-cornered behavior. These jumps, which are very important for vacancy drag, are therefore determined by a complex balance between SP properties and binding energies.

\section{Attempt frequencies}

The solute attempt frequencies (for the $\omega_{2}$ jump) are shown in Fig. 4. The results refer to calculations in 53-atom cells only because of the high computational cost required. However, the attempt frequencies for $\mathrm{Fe}$ and $\mathrm{Al}$ were computed in 127atom cells as well. It was found that in both cases the attempt frequency in the bigger simulation cell is about $10 \%$ larger. For this reason, the values shown in Fig. 4 are considered to be affected by an uncertainty of $\pm 10 \%$.

The shapes of the group trends are again similar to those found previously for binding energies and migration barriers, although the behavior is here more irregular. The $3 d$ row is $\mathrm{M}$ shaped once again because of the odd behavior of $\mathrm{Mn}$, whereas the expected parabolic behavior of the other rows is disturbed by the uncertainty in the attempt frequency calculations $(5 d)$ and the out-of-trend value of $\mathrm{Ru}(4 d)$.

In general, it is evident that solutes with a low migration barrier are also characterized by a lower attempt frequency. 


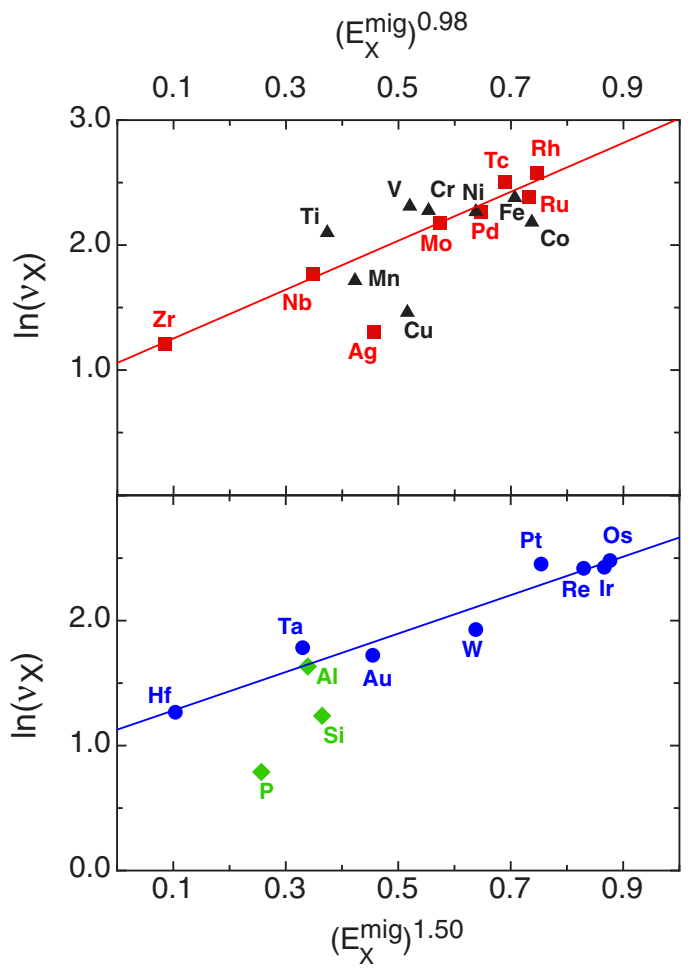

FIG. 6. Meyer-Neldel plot of solute migration energies versus attempt frequencies (jump $\omega_{2}$ ) for $3 d$ and $4 d$ metals (above), and $5 d$ metals and $3 p$ elements (below). The fitting lines for the $4 d$ and $5 d$ elements are obtained with the least-square method.

This compensation effect is in qualitative agreement with the Meyer-Neldel rule (MNR), which was reported for the first time to describe conduction processes in disordered materials [62], and has thereafter been observed for several thermally activated processes, not limited to condensedmatter physics [63]. For what concerns diffusion phenomena, the MNR has been successfully applied to surface selfdiffusion [64] and adatom diffusion [65] in $\mathrm{Cu}$. In both cases, the activation energies and the prefactors were obtained by means of molecular dynamics simulations and were found to be linked by the following relationship:

$$
v_{x}=v_{0} \exp \left(\frac{E_{x}^{\mathrm{mig}}}{\epsilon_{0}}\right)^{\alpha}
$$

where the process-dependent constants $\nu_{0}$ and $\epsilon_{0}$, as well as the exponent $\alpha$, were used as fitting parameters. $\epsilon_{0}$ is also referred to as the Meyer-Neldel energy (MNE) [63]. Hence, according to the MNR, when plotting $\left(E^{\text {mig }}\right)^{\alpha}$ versus $\ln (v)$, the data should lie on a straight line. For migration processes, the exponent $\alpha$ determines what types of phonons are involved. According to some phenomenological models [66,67], an exponent $\alpha$ between 0.5 and 1 implies the main contribution by acoustic phonons.

It is therefore interesting to investigate if solute migration in an iron matrix can be also quantitatively described by Eq. (14). The fitting could not be performed on the whole set of data, but it was at any rate successful on the single rows individually. The fitting results are shown in Fig. 6. An exponent $\alpha=0.98$ was found to fit best the $4 d$ elements and some of the $3 d$ elements, whereas $\alpha=1.5$ was found to describe well the $5 d$ row. The fitting could not be performed on the $3 p$ elements because of the limited amount of data points. The fitting led to the same $v_{0}=3 \mathrm{THz}$ for both rows, but with a different MNE, namely, $\epsilon_{0}=0.52 \mathrm{eV}(4 d)$ and $\epsilon_{0}=0.73 \mathrm{eV}(5 d)$. Such values are considerably higher than the Debye temperature in iron $(0.04 \mathrm{eV})$, and also larger than what was reported by previous works $\left(\epsilon_{0}=0.074 \mathrm{eV}\right)[64,65]$. In addition, the difference is similar to the difference in solute migration energy between the $4 d$ and $5 d$ elements. In some models [63], the ratio $E^{\mathrm{mig}} / \epsilon_{0}$ is claimed to be related to the mean number of excitations that are necessary for a solute jump. Here, this quantity is seemingly constant between the $4 d$ and $5 d$ series. Finally, the noble metals $\mathrm{Ag}$ and $\mathrm{Cu}$ are found to lie out of the trend (the $4 d$ fitting was performed by neglecting the $\mathrm{Ag}$ data point). Therefore, it can be concluded that this set of data can be also quantitatively fitted into the MNR, although with some exceptions, in particular the $3 d$ elements and the noble metals. The higher exponents found here with respect to the $\mathrm{Cu}$ diffusion studies $[64,65]$ might indicate that solute diffusion is actually guided by local optical modes, which are strongly related to the solute-vacancy chemical bonding.

\section{E. Solute-vacancy drag}

The vacancy-drag tendencies as functions of temperature are shown in Fig. 7(a). The wind factor $L_{x \mathrm{v}} / L_{x x}$ is positive when drag occurs, and negative otherwise. As was already highlighted for some impurities [15], all curves are S shaped, going from vacancy drag at low temperature to the inverse Kirkendall mechanism at high temperature. The flux-coupling strength determines the transition temperature, shifting the curve along the temperature axis.

It is possible to observe a strong correlation between drag tendencies and $1 \mathrm{nn}$ and $2 \mathrm{nn}$ binding energies. Solutes with $1 \mathrm{nn}$ and $2 \mathrm{nn}$ attractive characters with vacancies are dragged up to higher temperatures, with a smooth transition; conversely, solutes with a $1 \mathrm{nn}$ binding interaction and a $2 \mathrm{nn}$ repulsive one are characterized by a sharper transition and a lower transition temperature. This proves that vacancy drag can occur also in presence of a repulsive $2 \mathrm{nn}$ interaction, contrary to previous statements [26,27], as a consequence of the combination of the $\omega_{i j}$ jump frequencies. In addition, vacancy drag does not occur for weakly interacting elements such as $\mathrm{Cr}$ and $\mathrm{V}$, whereas $\mathrm{Co}$ is dragged below $450 \mathrm{~K}$, in spite of the absence of a $1 \mathrm{nn}$ binding interaction. Hence, vacancy drag, although minimal, can occur even thanks to attractive $2 \mathrm{nn}$ interactions only. It is possible therefore to conclude that the $1 \mathrm{nn}$ binding interaction is surely the dominant factor, if present, but does not represent a necessary condition for vacancy drag. Conversely, a binding $2 \mathrm{nn}$ interaction can considerably extend the vacancy drag range, but a repulsive one is definitely negligible if paired with an attractive $1 \mathrm{nn}$ one.

For the $4 d$ and $5 d$ elements, the curves of Fig. 7(a) are coupled in order to show that elements with the same number of electrons in the outermost $d$ band are also characterized by very similar flux-coupling behaviors, thanks to the similar binding-energy trends. Drag is systematically stronger for the $4 d$ metals because of the stronger $1 \mathrm{nn}$ binding. It is worthwhile noticing that the drag tendency is completely independent from 
(a) Vacancy drag

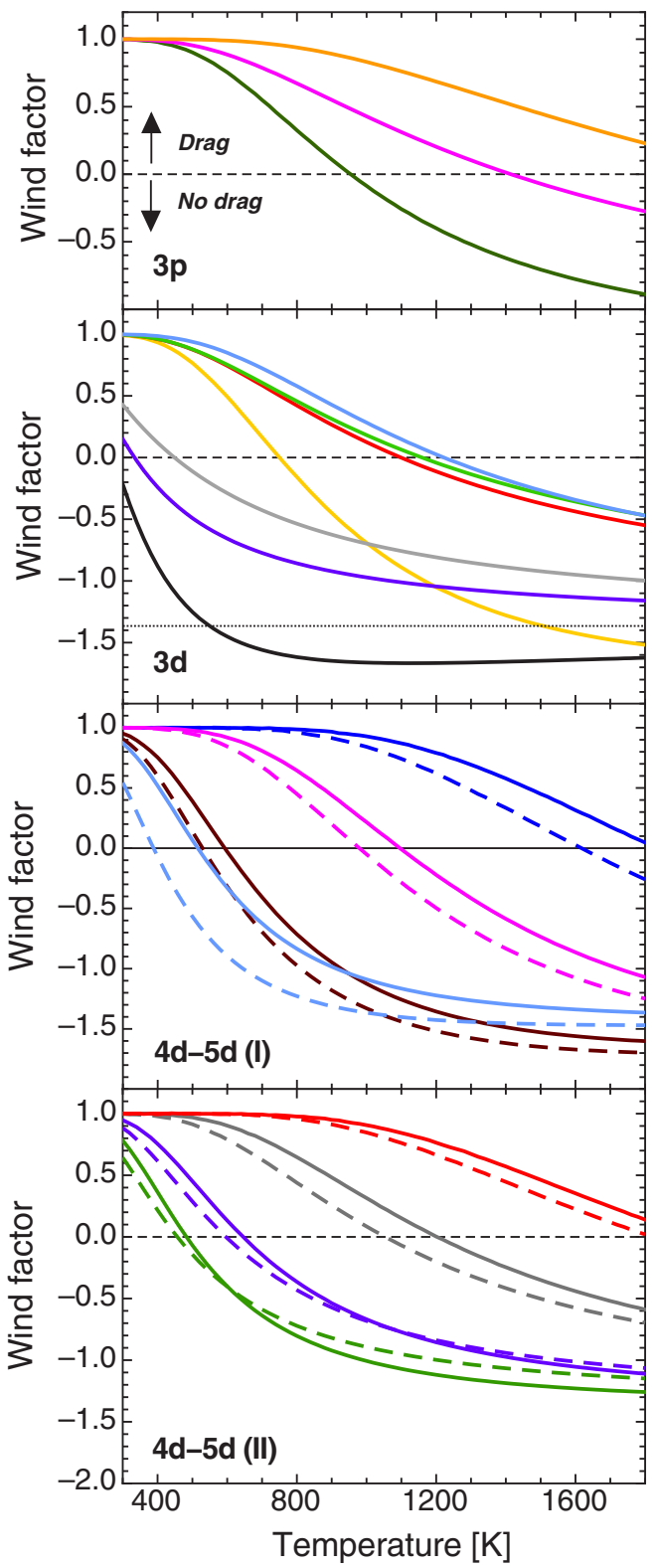

(b) RIS tendencies

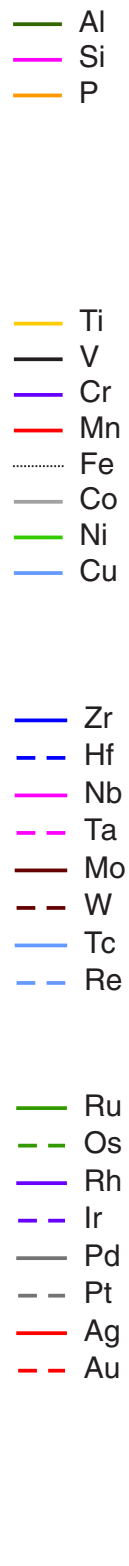

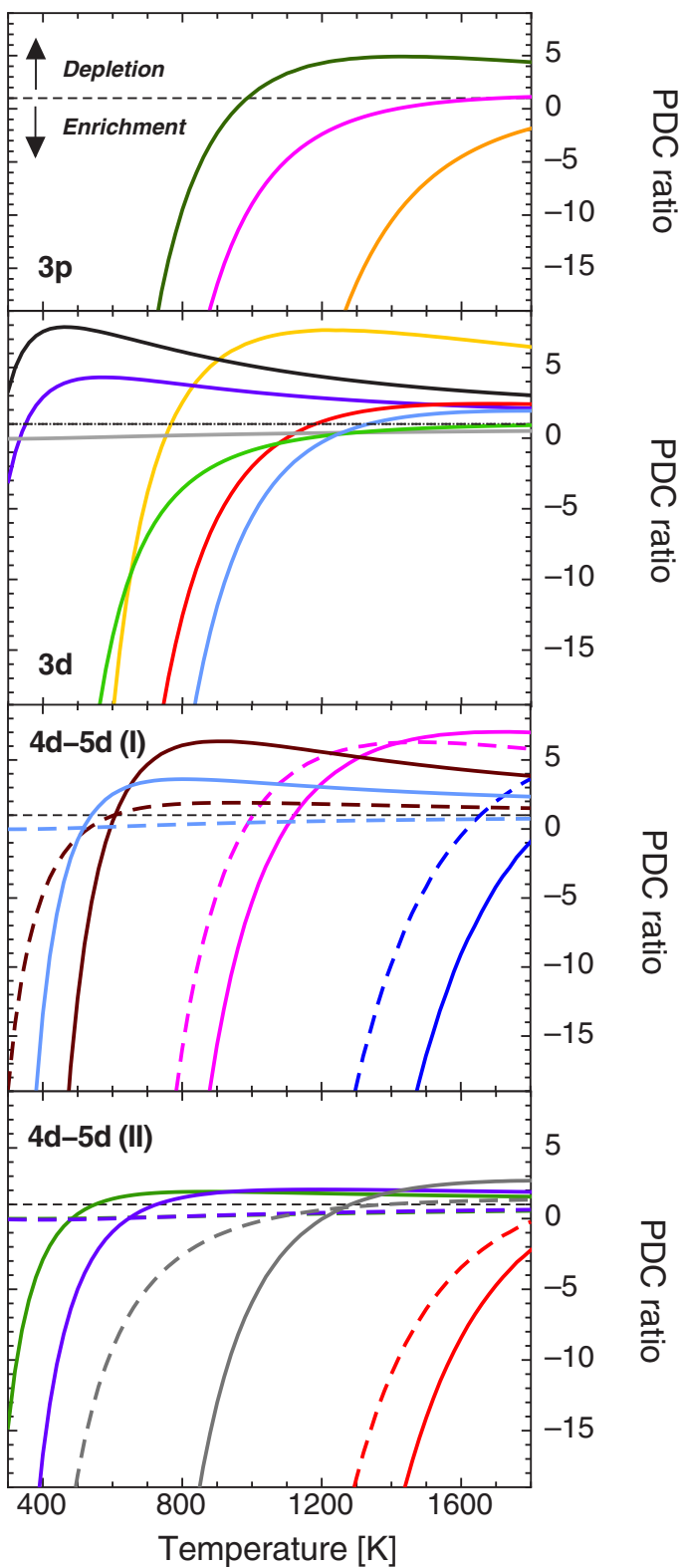

FIG. 7. (a) Solute-vacancy drag tendencies as functions of temperature for the $\mathrm{Fe}(\mathrm{x})$ dilute binary alloys. Drag occurs for positive values of the wind factor. (b) Ratio of solute-to-iron partial diffusion coefficients (PDC) as defined in Eq. (3). Solute enrichment (via vacancy mechanism) occurs for values of this ratio smaller than unity (the solute is slower than Fe) or negative (vacancy drag). The lines of Ir and Os are perfectly superposed.

the solute jump frequency. It is also necessary to point out that the (already strong) drag tendency of the band-end elements is most likely underestimated because of the $5 \mathrm{nn}$ solute-vacancy interaction, which is neglected in this SCMF model.

The group trends of the wind factor at $600 \mathrm{~K}$ and of the transition temperature are shown in Figs. 8(a) and 8(c), respectively. Because of the strong correlation with the binding energy, the trends are very similar: a parabolic shape for the $4 d$ and $5 d$ TM's, and a W-shaped curve for the $3 d$ metals. Flux coupling for $\mathrm{Mn}$ is considerably stronger than its neighbors on the periodic table. The only exception is represented by $\mathrm{Cu}$, for which the transition temperature is markedly lower than the other noble metals. Conversely, the neighboring solute $(\mathrm{Ni})$ matches the drag tendency of the corresponding metals $(\mathrm{Pd}$ and $\mathrm{Pt}$ ). This is a direct consequence of the anomaly observed in the $2 \mathrm{nn}$ binding energy for the noble metals (Fig. 3), which is reflected onto a lower $\omega_{24}$ barrier (Fig. 5). This anomaly implies a higher escape frequency from $2 \mathrm{nn}$ in $\mathrm{FeCu}$, and therefore a weaker drag tendency. In a previous study [15], this anomaly was erroneously ascribed to the stronger Ni-vacancy $2 \mathrm{nn}$ interaction with respect to the $1 \mathrm{nn}$ one, but the systematic approach of this work clarifies that the exception is actually represented by $\mathrm{Cu}$. It is also remarkable that a seemingly small difference in the $\omega_{24}$ frequency, which is often disregarded in 
(a) Wind factor at $600 \mathrm{~K}$

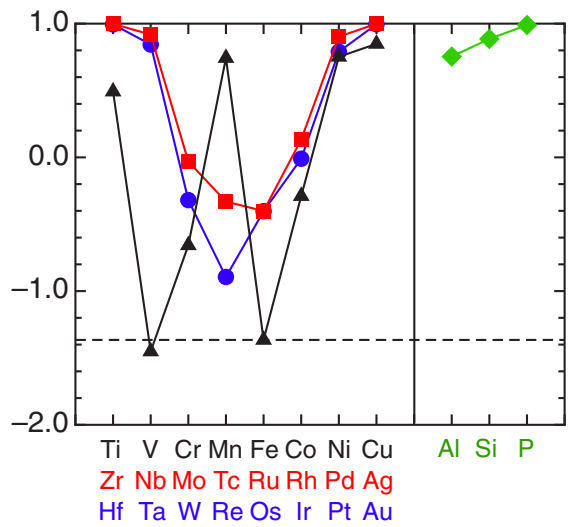

(d) Diffusion activation energy [eV]

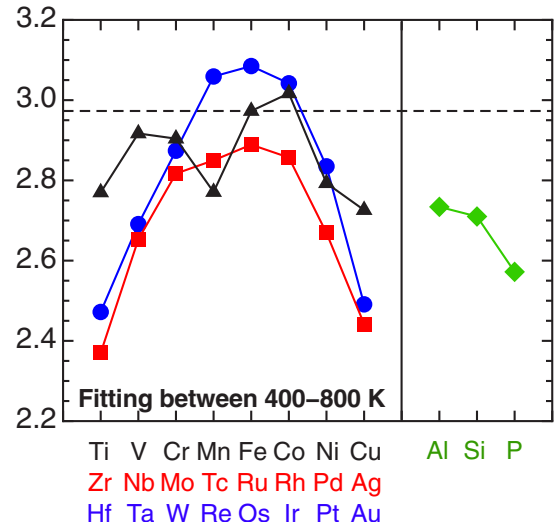

(b) Correlation factor at $600 \mathrm{~K}$

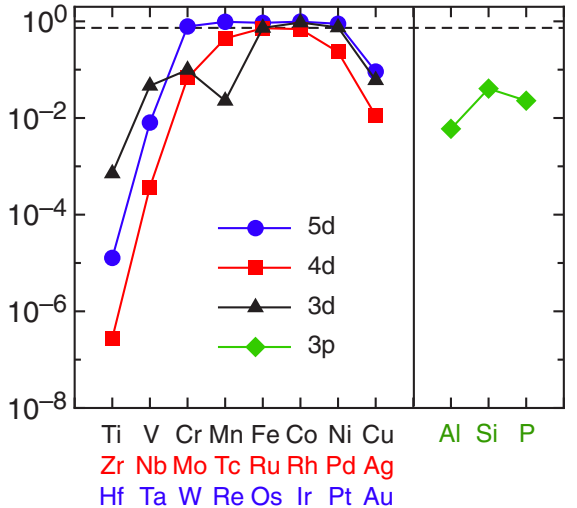

(e) Diffusion prefactor $\left[\mathrm{cm}^{2} / \mathrm{s}\right]$

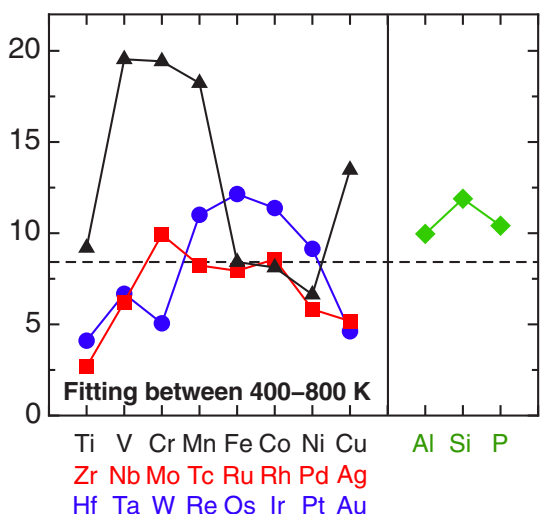

(c) Drag transition temperature [K]

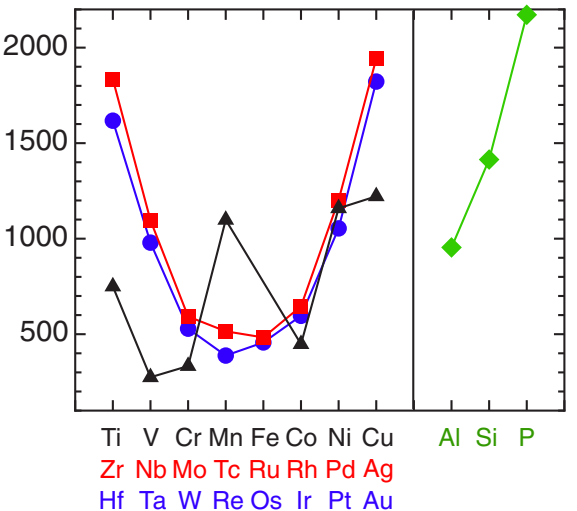

(f) RIS switchover temperature [K]

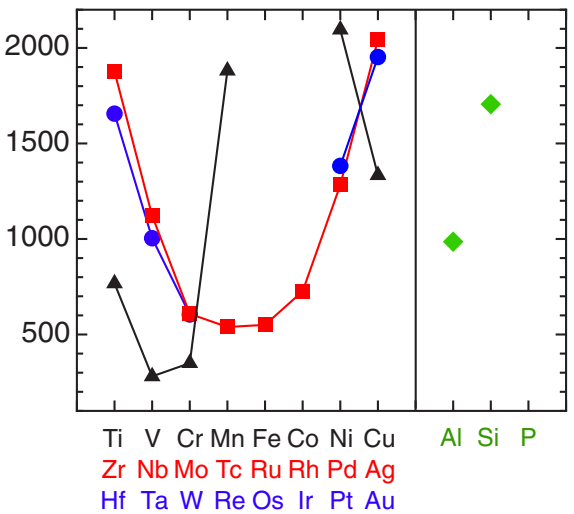

FIG. 8. Group trends of (a) the wind factor at $600 \mathrm{~K}$, (b) the solute-diffusion correlation factor at $600 \mathrm{~K}$, (d) the solute-diffusion activation energy, (e) prefactors corresponding to a least-square fitting between 400 and $800 \mathrm{~K}$, (c) the transition temperature from drag to nondrag tendencies, and (f) from RIS enrichment to depletion tendencies. In the latter, the elements with a temperature-independent enrichment tendency are not shown. The dashed lines represent the values for pure Fe.

flux-coupling studies, has such a large effect on the $\mathrm{Cu}$ drag transition temperature $(600 \mathrm{~K}$ lower than $\mathrm{Ag}, \mathrm{Au})$.

The effect of the $2 \mathrm{nn}$ binding is visible in Fig. 8(c), as the transition temperatures are slightly higher for the late TM's than the early ones. In order to take into account the effect of both the $2 \mathrm{nn}$ binding energy and the saddle point, this asymmetry is discussed in terms of a combination of jump frequencies. In first approximation, the drag tendency depends on the ratio $\omega_{12} /\left(\omega_{13}+\omega_{15}\right)$, as $\omega_{12}$ allows the vacancy to turn around the solute, whereas $\omega_{13}$ and $\omega_{15}$ cause the pair to dissociate. It can be observed from Fig. 5 that such ratio is lower for the early TM's and higher for the late ones.

In conclusion, vacancy drag is the most common solutediffusion mechanism at low temperatures for all impurities except $\mathrm{Cr}$ and $\mathrm{V}$. Any low-temperature model assuming an inverse Kirkendall mechanism and neglecting flux coupling is therefore likely not to yield the correct transport properties. The found strong correlation with $1 \mathrm{nn}$ and $2 \mathrm{nn}$ interaction energies confirms that drag tendencies can indeed by qualitatively inferred from such energies, although the Onsager matrix must be always calculated in order to determine the strength and extent of vacancy drag. However, transport properties are in first place determined by kinetics, or in other words by the saddle-point properties. It was shown in Fig. 5 that such properties show the same trends as the binding energies, which justifies why it is possible to correlate the flux-coupling tendencies with thermodynamic interactions. Moreover, given the electronic origin of both trends that was discussed in the previous sections, it is also expected to find similar vacancydrag tendencies in other bcc transition metals, confirming that vacancy drag might be a widespread phenomenon not limited only to iron-based dilute alloys.

\section{F. RIS tendencies}

In irradiated materials, flux coupling can produce RIS at defect sinks because of the permanent production of defects. When vacancy drag occurs, vacancies diffusing to sinks carry solute atoms, causing the solute species to enrich for instance on grain boundaries or dislocations [35]. In the absence of drag, instead, the solute and matrix atoms diffuse away from the sink surface at a relative speed given by the PDC ratio [Eq. (3)]: if this ratio is smaller than 1, solute atoms are slower and enrichment still occurs, whereas depletion is observed in the opposite case.

The RIS tendencies due to vacancy diffusion only (thus assuming a neutral contribution from SIA's and neglecting the prefactor of Eq. (4)) are shown in Fig. 7(b) as functions 
of temperature. It is possible to distinguish two types of behavior. Most elements switch from an enrichment tendency due to drag at low temperature, to a depletion tendency in the high-temperature range, passing through a short interval of enrichment without drag. This is not uncommon: switch of RIS tendency from enrichment to depletion as a function of temperature has been observed, for instance, in $\mathrm{FeCr}$ alloys [5]. On the other hand, a few elements, namely, Co, Re, Os, and Ir, are slower than $\mathrm{Fe}$ at any temperature and hence enrichment always occurs, independently from the flux-coupling sign. Such elements are the "slow diffusers" in Fe, and the reason for their behavior is discussed in the next section.

For the "fast diffusers," the trends are obviously correlated to vacancy drag. The early TM's, with a repulsive $2 \mathrm{nn}$ interaction, are characterized by a sharp transition from enrichment to depletion, whereas the late TM's have a smoother transition and a higher switchover temperature. The weakly interacting elements such as $\mathrm{Cr}$ and $\mathrm{V}$ deplete at all temperatures because of the absence of drag. An analogous shift between corresponding $4 d$ and $5 d$ metals can be observed, for the same reason that was mentioned for the drag tendency.

The switchover temperature as a function of the $d$-band filling is shown in Fig. 8(f). Elements that are consistently enriched are not shown (for $\mathrm{P}$ the switchover temperature is very high). In spite of the missing elements, it is possible to recognize the same curve shapes, which are closely related to the vacancy drag trends of Fig. 8(c). Again, $\mathrm{Cu}$ has an exceptionally low critical temperature because of the same aforementioned anomalies. On the other hand, the critical temperature for $\mathrm{Ni}$ is much higher than the corresponding metals $(\mathrm{Pd}, \mathrm{Pt})$, in spite of comparable drag tendencies. This is related to a nontrivial combination of the $\omega_{i j}$ frequencies, which highlights the importance of kinetics in solute-diffusion modeling.

The outcome of this flux-coupling study in model dilute alloys can be qualitatively discussed in regard to several observations of solute transport in irradiated ferritic and $\mathrm{F} / \mathrm{M}$ alloys, neglecting any multisolute interactions that are likely to occur in real multicomponent and nondilute alloys. Vacancy drag can explain the nucleation of solute clusters containing $\mathrm{Mn}, \mathrm{Ni}$ on preexisting $\mathrm{Cu}$ clusters [8,68], interstitial loops [3,69,70], and dislocations [71], as well as the formation of Cu-vacancy clusters [72]. In RPV surveillance materials, precipitation of impurities such as $\mathrm{Mn}, \mathrm{Ni}, \mathrm{Si}, \mathrm{Cu}$, and $\mathrm{P}$ was observed with atom-probe tomography [8], and can also be explained by vacancies dragging solute atoms towards small sinks such as invisible interstitial loops, as suggested in [3,11]. In these clusters, however, no Mo is found, in spite of its nonnegligible concentration. This work shows that the drag transition temperature of Mo $(593 \mathrm{~K})$ is very close to that of RPV operation $(573 \mathrm{~K})$, hence confirming the weakness of the flux coupling with respect to the other mentioned impurities. In addition, several experiments have shown the segregation of $\mathrm{P}[8,71]$ and $\mathrm{Mn}$ [70] on grain boundaries and dislocations, as well as the enrichment profiles at grain boundaries of other elements such as $\mathrm{Si}, \mathrm{Ni}, \mathrm{Cu}$, and $\mathrm{Cr}$ [5]. The Cr RIS tendency cannot be comprehensively discussed here because of the stability of the Fe-Cr mixed dumbbell, which suggests that the interstitial contribution to the RIS tendency cannot be neglected. As for $\mathrm{Si}, \mathrm{Ni}$, and $\mathrm{Cu}$, the bell-shaped enrichment tendencies observed in [5] as functions of temperature are compatible with the trends of Fig. 7(b). At low temperature, there should be strong vacancy drag and therefore a marked enrichment tendency according to this work's model; however, at such temperatures defects are very slow, and defect recombination is enhanced. Therefore, even in steady-state conditions, no RIS occurs and no change of solute concentration is found. With increasing temperature, the enrichment tendency decreases due to the PDC ratio, whereas defect mobility is enhanced, consistently with the experimental observations. However, this represents only a secondary effect, as RIS is mostly dampened and eventually stopped by the vanishing difference between point defect saturation and the corresponding equilibrium values.

\section{G. Solute-vacancy correlations}

The solute correlation factor $f_{x}$ appearing in Eq. (6) accounts for the solute "slowing down" due to the probability of performing two consecutive exchanges with a neighboring vacancy (forward and backward), which do not contribute to an effective displacement of the solute atom. In a noninteracting bcc alloy (or in a pure bcc metal), this corresponds to the geometric factor $f_{0}=0.727$ [17]. $f_{x}$ can range from 0 to 1 , and tends to $f_{0}$ as temperature increases, since correlations are smoothened by the Boltzmann factor $1 / k_{B} T$.

The correlation factors are here obtained by means of Eq. (A1) and are shown for each solute group in Fig. 8(b) at $600 \mathrm{~K}$. The temperature-dependent factors are provided for each solute in the Supplemental Material [73]. It should be pointed out that the correction provided by Eq. (A1) with respect to the previously available formula [17] does not significantly affect the value of the solute-diffusion coefficients.

Once again, the group trends are $\mathrm{M}$ shaped for the $3 d$ metals and bell shaped for the $4 d$ and $5 d$ metals. The top of the curves is flat because the correlation factor cannot exceed 1 . These trends are clearly correlated to the solute migration barrier, which is characterized by the same shape and the same type of asymmetry. The very low migration barriers of the early TM's make the correlation factor (and hence the solute-diffusion coefficient) decrease by several orders of magnitude, and this effect grows stronger at low temperatures, up to $10^{-13}$ for $\mathrm{Zr}$ at $300 \mathrm{~K}$. The compensation provided by the solute attempt frequency is secondary, and negligible at low temperatures. Solute-vacancy correlations can therefore make solute diffusion considerably slower, as the solute can effectively "trap" the vacancy in a $1 \mathrm{nn}$ position. Moreover, they introduce deviations from the Arrhenius behavior at low temperatures, contributing to the inaccuracy of extrapolations from high-temperature diffusion data.

This qualitative description can be completed by comparing $\omega_{2}$ with the other jump frequencies. The correlation strength can be connected with the probability of the complementary jumps to $\omega_{2}$, namely, the jumps leading to a dissociation of the solute-vacancy pair at $1 \mathrm{nn}$. One can define an average dissociation frequency $\omega_{1 *}$ as

$$
\omega_{1 *}=\frac{1}{7}\left(3 \omega_{12}+3 \omega_{13}+\omega_{15}\right),
$$

where the average is weighed upon the available dissociation paths. The ratio $\omega_{1 *} / \omega_{2}$ represents therefore the probability 


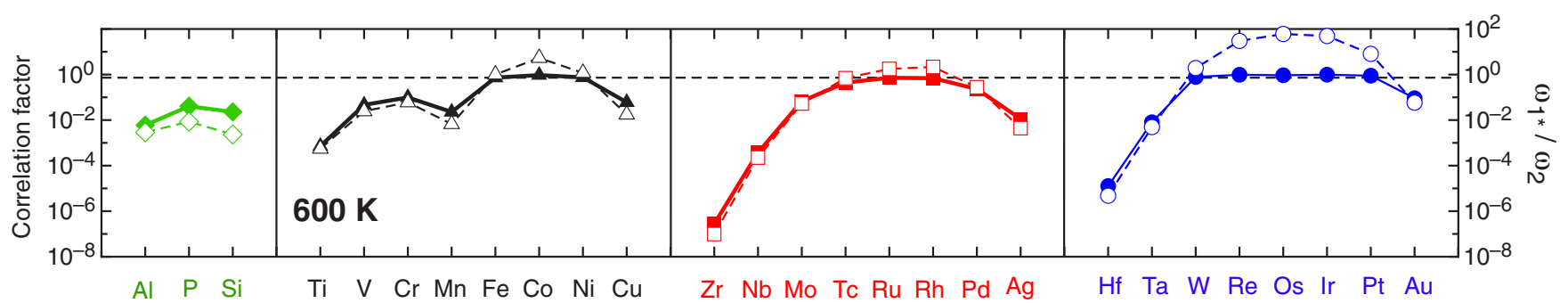

FIG. 9. Solute-diffusion correlation factors (full symbols) and ratio of average $1 \mathrm{nn}$ dissociation frequency $\omega_{i j}$ to solute jump frequency $\omega_{2}$ (open symbols) at $600 \mathrm{~K}$. The correlation factor cannot assume values larger than 1 . The dashed line marks the correlation factor value in pure $\mathrm{Fe}\left(f_{0}=0.727\right)$.

of escaping the $1 \mathrm{nn}$ configuration and allowing the vacancy to access the paths around the solute atom to produce a net solute displacement. In Fig. 9, the calculated correlation factors at $600 \mathrm{~K}$ are compared to the $\omega_{1 *} / \omega_{2}$ ratio at the same temperature. The astounding match between the two highlights that solute-vacancy correlations are indeed controlled by this frequency ratio, in accordance to Manning's early formulation of $f_{x}$ [74]. For the early TM's, hence, the strong correlation is not determined only by the low migration barrier, but is enhanced by the high barriers for the $\omega_{12}$ and $\omega_{13}$ jumps, which is not the case for the late TM's. It can be therefore concluded that solute-vacancy correlations are stronger for the band-end elements, and particularly strong for the early ones, because of the combined effect of low solute migration barriers, high $1 \mathrm{nn}$ solute-vacancy binding energy, and the saddle-point electronic interactions.

With the same argument, it is possible to explain why some elements, such as $\mathrm{Co}, \mathrm{Ni}$, and the middle-row elements of the $4 d$ and $5 d$ bands, show a "negative correlation," i.e., their correlation factor is above the geometric factor $f_{0}$. For these elements, the $\omega_{1 *} / \omega_{2}$ ratio is larger than 1 , which entails that in the $1 \mathrm{nn}$ configuration the vacancy has a larger probability to escape than to exchange with the solute. Hence, the correlation factor approaches 1 because the vacancy tends to "avoid" the solute, or anyway not to undergo repeated exchanges. This efficiency of the diffusion process from a kinetic standpoint is anyway counterbalanced by thermodynamics since for these elements, the $1 \mathrm{nn}$ binding energy is small and thus so is the probability to have a vacancy next to the solute, which is a necessary condition for solute diffusion to occur. On the other hand, for the strongly correlated solutes the $\omega_{1 *} / \omega_{2}$ ratio is very small and the vacancy does not have the possibility to leave the solute, therefore impeding solute diffusion because the vacancy is trapped and cannot move.

\section{H. Solute-diffusion coefficients}

The self-diffusion and solute-diffusion coefficients, obtained by means of Eq. (5), are shown, respectively, in Figs. 10 and 11 for the impurities for which experimental measurements are available, and are compared with experimental measurements [32,75,76] and previous calculations [21-24]. For the sake of consistency with the solute attempt-frequency calculations, the diffusion coefficients are calculated with the values for $S_{\mathrm{v}}^{\mathrm{f}}$ and $\nu_{\mathrm{Fe}}$ obtained in 53-atom cells. Each diffusion coefficient is corrected with the magnetic factor of Fig. 2, in order to account for the effects of the FM-to-PM transition.
Because of the magnetic-related non-Arrhenius behavior, the fitting in the FM region is extremely sensitive to the chosen temperature range. In order to provide values of $Q$ and $D_{0}$ for low-temperature applications, the fitting results in the temperature range $400-800 \mathrm{~K}$ are reported in Fig. 8(d) $(Q)$ and $8(\mathrm{e})\left(D_{0}\right)$. The numerical values of $Q$ and $D_{0}$, as well as the solute-diffusion coefficients in the temperature range $800-1800 \mathrm{~K}$ for all solutes are to be found in the Supplemental Material [73].

There is an overall satisfactory agreement with experiments. The perfect match with self-diffusion measurements proves the quality of the magnetic model, which did not entail any fitting procedure. The solute-diffusion coefficients are also well matched with experiments, for what concerns the activation energy $Q$ (i.e., the slope in the Arrhenius plot) and the change of slope across the Curie temperature, with the exception of $\mathrm{Mn}$. The accuracy in the $Q$ value confirms the reliability of this model in the prediction of low-temperature solute-diffusion coefficients. Moreover, the comparison with previous calculations in iron with $\mathrm{Al}$ [23], $\mathrm{P}$ [21], $\mathrm{Cu}$ [22], Mo [24], and W [24] solute atoms shows that the values obtained in this work are always in better agreement with experiments, although the difference with the calculations in [24] is minimal. The mismatch in the Mn diffusion coefficient makes the extrapolation to low

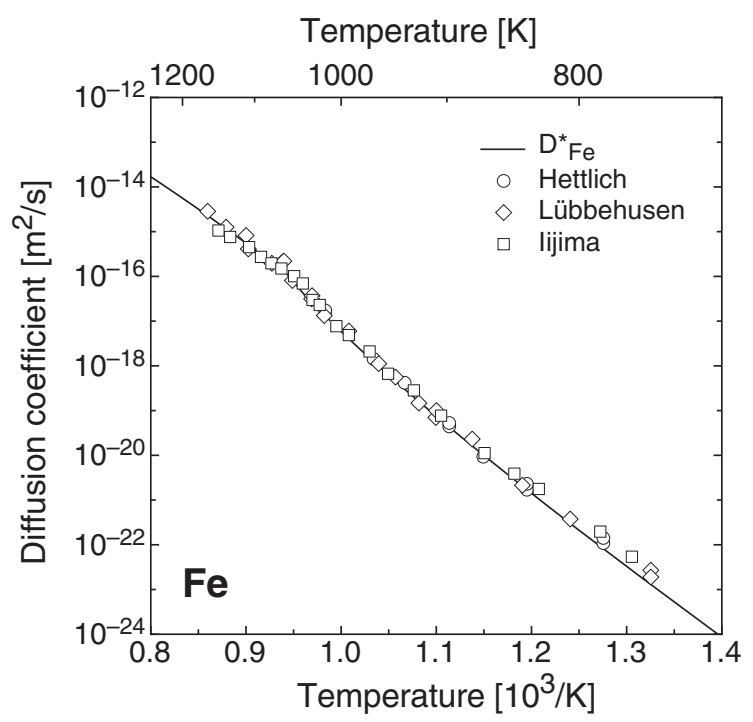

FIG. 10. Tracer self-diffusion coefficient calculated in this work, and comparison to experimental measurements [76]. 


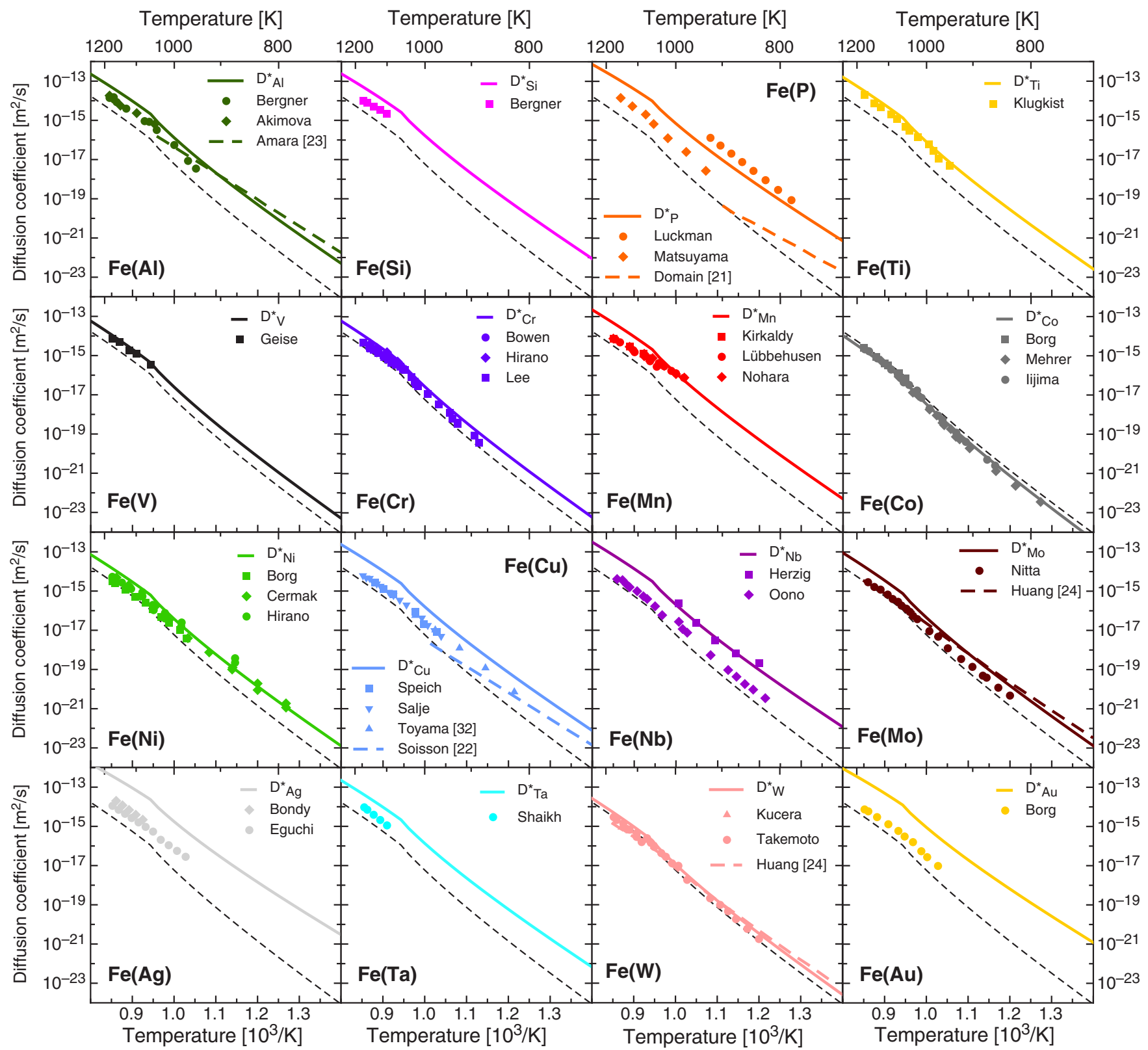

FIG. 11. Solute tracer diffusion coefficients calculated in this work, and comparison to experimental measurements [32,75,76] and previous calculations [21-24]. The dashed lines mark the Fe tracer self-diffusion coefficient, as shown in Fig. 10.

temperatures unreliable, and shows the inadequacy of the pure-Fe magnetic model for this peculiar system. In order to properly describe solute diffusion across the Curie temperature, the direct effect of magnetism on the solute PM activation energy should be carefully modeled, hence calling for further investigations of the Mn solute magnetic behavior in $\mathrm{Fe}$.

On the other hand, for a few solutes the agreement in the diffusion prefactor is not as satisfactory. The prefactor is consistently overestimated, although the difference is in most cases limited, and it does not exceed one order of magnitude, approximately. In Fig. 12, the diffusion coefficients here calculated are compared to experimental measurements at $1100 \mathrm{~K}$. It can be observed that the mismatch is small for the middle TM's (except $\mathrm{Mn}$ ) and progressively increases towards the band ends, which might suggest the presence of a systematic error. This error might be due to the assumption $v_{i j}=v_{\mathrm{Fe}}$ for all iron jumps, or to the inadequacy of a $2 \mathrm{nn}$ thermodynamic model for the large-sized solutes, which are characterized by a nonnegligible $5 \mathrm{nn}$ interaction. It is also worthwhile mentioning that some entropic contributions, such as the binding entropy or the electronic entropy, are here neglected. The effect of lattice expansion at nonzero temperature might also play a role, although this effect would be temperature dependent. Since the mismatch affects the prefactor only, the accuracy of the calculated solute-diffusion coefficients at any temperature can be improved by simply shifting the curve down by the mismatch factor, inferable for instance from Fig. 12. The mismatch values can also be found in the Supplemental Material [73]. 


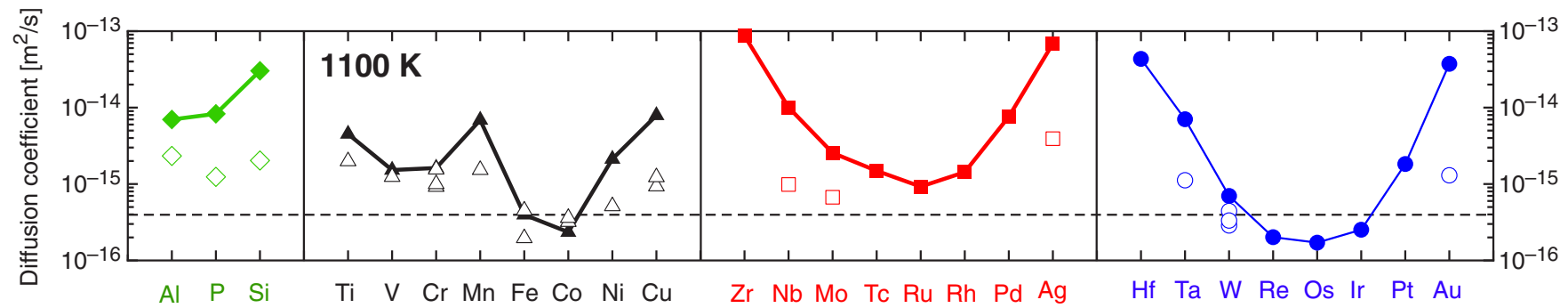

FIG. 12. Solute tracer diffusion coefficients at $1100 \mathrm{~K}$, as calculated in this work (full symbols) and from experimental measurements (open symbols) $[32,75,76]$.

The activation energies shown in Fig. 8(d) follow the same group trends observed for many other quantities in this work. These trends, which are also visible in the experimental coefficients of Fig. 12, are analogous to those obtained for solute diffusion in $\mathrm{Ni}$ [30] and $\mathrm{Al}$ [60]. Since they are mainly determined by the solute migration barrier trends of Fig. 4, it is possible to state that the activation energy for solute diffusion is controlled by the saddle-point interactions discussed in the previous section. However, the $Q$ curve is more symmetric than $E^{\mathrm{mig}}$ because of the compensation effect given by the correlation factor, and to a smaller extent by the attempt frequency, in accordance with the MNR. As previously discussed, solute-vacancy correlations are stronger for the early TM's and reduce the diffusion coefficient by several orders of magnitude, especially at low temperature. As a consequence, the effective activation energy of the late TM's is comparable to that of the early TM's.

On the contrary, the $D_{0}$ trends are not trivial to explain, as they are affected by $f_{x}$ and the less smooth behavior of the solute attempt frequency [Fig. 4(b)]. However, the usual M-shaped and bell-shaped trends can be obtained by applying the aforesaid correction to the prefactor, based on the mismatch with the experiments (under the assumption that the mismatch is bell shaped also for solutes with missing experimental measurements).

Among all TM impurities, Co is the only one known to be a slower diffuser than Fe. This exception, which is well caught by the calculations in this work, might be mistakenly ascribed to the larger migration barrier of $\mathrm{Co}(0.73 \mathrm{eV})$ with respect to $\mathrm{Fe}(0.70 \mathrm{eV})$. However, this is also the case for other impurities such as Ru, Rh, W, Re, Os, Ir, and Pt (Fig. 4). It was suggested that such behavior might be due to kinetic correlations [57]. This can be checked by analyzing the ratio of solute-diffusion to self-diffusion coefficient [36]:

$$
\frac{D_{x}^{*}}{D_{\mathrm{Fe}}^{*}}=\frac{f_{x}}{f_{0}} \frac{v_{x}}{v_{0}} \exp \left[-\frac{\left(\Delta E^{\mathrm{mig}}+E_{1 \mathrm{nn}}^{\mathrm{b}}\right)}{k_{B} T}\right],
$$

where $\Delta E^{\mathrm{mig}}=E_{2}^{\mathrm{mig}}-E_{0}^{\mathrm{mig}}$. Since the solute attempt frequencies are not very different from $v_{\mathrm{Fe}}$, the $D_{x}^{*} / D_{\mathrm{Fe}}^{*}$ ratio is controlled by the exponential term and the correlation factor $f_{x}$. Moreover, since $f_{x}<1$, the exponential term dominates if $f_{x}$ is larger than $f_{0}$. Since the correlation factor for all the aforementioned impurities is close to unity, the exponential term is dominant and kinetic correlations play a minor role. Then, the ratio of Eq. (16) is lower than 1 if $\Delta E^{\mathrm{mig}}>-E_{1 \mathrm{nn}}^{\mathrm{b}}$. In other words, the $1 \mathrm{nn}$ binding energy counteracts the higher solute migration barrier because it increases the probability of having a vacancy next to the solute. Therefore, $\mathrm{Ru}, \mathrm{Rh}, \mathrm{W}$, and $\mathrm{Pt}$ are faster diffusers than Fe because their $1 \mathrm{nn}$ binding energy is larger than $\Delta E^{\mathrm{mig}}$, as is confirmed by experiments for W. On the other hand, Co is a slow diffuser because the $1 \mathrm{nn}$ interaction is very weakly repulsive. Therefore, the low Co diffusivity is due to the (missing) thermodynamic interaction with a vacancy at $1 \mathrm{nn}$ rather than to kinetic correlations. Analogously, this model predicts that Re, Os, and Ir are slow diffusers as well, although no experiments have been yet performed to confirm this prediction. The method proposed in this work is therefore useful to predict the diffusion coefficients of impurities for which experimental measurements are missing, and to provide such quantities in low-temperature ranges that are not accessible by experiments.

\section{CONCLUSIONS}

The aim of this work was to investigate impurity diffusion in bcc iron with a systematic approach to all the $3 d, 4 d$, and $5 d$ solutes, as well as $\mathrm{Al}, \mathrm{Si}$, and $\mathrm{P}$. This was achieved by combining electronic-structure calculations of solute-vacancy interactions, jump frequencies, and attempt frequencies with an accurate mean-field computation of transport coefficients. From the latter were inferred solute-vacancy flux-coupling tendencies, correlations, and diffusion coefficients.

Remarkably, the variation of almost all properties with respect to the $d$-band filling follows similar trends: the properties of $4 d$ and $5 d$ impurities are bell shaped, whereas $3 d$ elements are influenced by magnetic interactions and show consistently an M- (or W-) shaped trend because of the peculiar behavior of Mn. Such common trends are all determined by the character of electronic interactions between the solute and the neighboring atoms next to a vacancy. Common size-factor (misfit-strain) arguments are shown to play only a minor role, in agreement with calculations in other matrices. The main exception to the general trends is represented by the $2 \mathrm{nn}$ binding energy, which varies linearly along the $d$ band. Similar interactions are also found at the saddle point of a migrating iron atom next to a solute. The migration barriers are therefore determined by the combination of saddle-point interactions and binding energies. Conversely, the solute migration barriers are governed mainly by the compressibility of the corresponding solid: large atoms are also more compressible and can hence migrate faster. However, this is partially compensated by two effects: a lower attempt frequency, in accordance with the Meyer-Neldel compensation law, and stronger correlations lowering the 
diffusion coefficient by several orders of magnitude. The combination of high migration barrier and low $1 \mathrm{nn}$ binding energy makes cobalt a "slow diffuser" in iron, and the same behavior is predicted for other impurities (namely Re, Os, and Ir), although experimental measurements for these solutes are still missing.

Vacancy drag is found to be the dominant flux-coupling mechanism at low temperature for almost all impurities. Contrary to common beliefs, drag can occur also for impurities with repulsive $2 \mathrm{nn}$ interactions with vacancies, as the $1 \mathrm{nn}$ binding energy is the dominant factor. The common origin of binding energies and saddle-point interactions allows the coupling tendencies to be qualitatively analyzed from bindingenergy trends only, although a quantitative analysis must rely on the transport-coefficient calculation. Finally, drag causes a clear solute-enrichment tendency at defect sinks, and a switchover to depletion across a large range of temperatures. Conclusions about RIS tendencies are to be corroborated by the analysis of dumbbell transport.

In conclusion, this systematic investigation has revealed the common electronic origins of flux-coupling and solutediffusion trends, contributing to a deeper understanding of impurity-diffusion mechanisms in metals and providing a reliable, $a b$ initio-based kinetic database of diffusion properties. Such common origins are seemingly solute dependent rather than matrix dependent, suggesting that similar trends might arise as well in other transition metals. This work has also yielded solute-diffusion coefficients in good agreement with experiments, providing ready-to-use low-temperature values that are not experimentally viable, and even for the missing impurities. The provided database of diffusion properties represents therefore a valuable contribution that can be employed in the modeling of a vast range of diffusion-controlled aging phenomena.

\section{ACKNOWLEDGMENTS}

This work has received funding from the Euratom research and training programme 2014-2018 under the Grant Agreement No. 661913 (SOTERIA). It contributes to the Joint Program on Nuclear Materials (JPNM) of the European Energy Research Alliance (EERA). This work was also funded by Vattenfall and Göran Gustafsson Stiftelse för Naturvetenskaplig och Medicinsk Forskning. The highperformance computing resources were provided by the Swedish National Infrastructure for Computing (SNIC). Furthermore, the authors acknowledge C. Marinica, F. Willaime, and B. Legrand for the fruitful discussions.

\section{APPENDIX A: CORRELATION FACTOR}

The correlation factor $f_{x}$ appearing in Eq. (6) can be expressed as a function of the atomic jump frequencies, in a multifrequency framework such as for instance the ones defined in [36]. Different expressions are available, depending on the extent of the solute-vacancy interactions and consequently of the amount of jump frequencies to be considered. The most complete one [17] includes $1 \mathrm{nn}$ and $2 \mathrm{nn}$ interactions, but was developed under the assumption $\omega_{42}=\omega_{0}$.

Thanks to the SCMF theory, it is possible to provide a more accurate expression of $f_{x}$ in a bcc dilute binary alloy with $1 \mathrm{nn}$ and $2 \mathrm{nn}$ solute-vacancy interactions, removing the $\omega_{42}=\omega_{0}$ assumption. It reads as

$$
f_{x}=1-\frac{2 \omega_{2}}{2 \omega_{2}+3 \omega_{12}+3 \omega_{13}+\omega_{15}-\frac{\omega_{12} \omega_{21}}{\omega_{21}+\omega_{24}}-\frac{\omega_{15} \omega_{51}}{\omega_{\mathrm{A}}}-\frac{\omega_{\mathrm{C} 1} \omega_{\mathrm{C} 2}}{\omega_{\mathrm{B}}}+\frac{2 \omega_{\mathrm{B}}\left(\omega_{13}+k \omega_{15}+\frac{\omega_{0} \omega_{\mathrm{C} 1}(1+k)}{\omega_{\mathrm{B}}}\right)\left(\omega_{31}+k \omega_{51}+\frac{\omega_{0} \omega_{\mathrm{C} 2}(1+k)}{\omega_{\mathrm{B}}}\right)}{\omega_{\mathrm{B}}\left(3 \omega_{0}+\omega_{31}-k \omega_{\mathrm{A}}-2 k^{2} \omega_{\mathrm{A}}\right)-2 \omega_{0}^{2}(1+k)^{2}}},
$$

where the following factors are introduced:

$$
\begin{aligned}
\omega_{\mathrm{A}} & =\omega_{51}+7 \omega_{0}-\frac{2 \omega_{0}^{2}}{\omega_{42}+7 \omega_{0}} ; \\
\omega_{\mathrm{B}} & =\omega_{42}+7 \omega_{0}-\frac{\omega_{24} \omega_{42}}{\omega_{21}+\omega_{24}}-\frac{\omega_{0}^{2}}{\omega_{\mathrm{A}}} ; \\
\omega_{\mathrm{C} 1} & =\frac{\omega_{12} \omega_{24}}{\omega_{21}+\omega_{24}}+\frac{\omega_{0} \omega_{15}}{\omega_{\mathrm{A}}} ; \\
\omega_{\mathrm{C} 2} & =\frac{\omega_{21} \omega_{42}}{\omega_{21}+\omega_{24}}+\frac{\omega_{0} \omega_{51}}{\omega_{\mathrm{A}}} \\
k & =\frac{\omega_{0}^{2}}{\omega_{\mathrm{A}}\left(\omega_{42}+7 \omega_{0}\right)} .
\end{aligned}
$$

The correction provided by this new expression is expected to be relevant when the association frequency $\omega_{42}$ is considerably different from $\omega_{0}$, which is not the case for the dilute alloys investigated in this work. At any rate, larger differences with respect to the previous Le Claire's formula [17] are also obtained at very low temperatures.

\section{APPENDIX B: MAGNETIC EXCESS ENTHALPY}

The magnetic excess enthalpy due to the magnetic transition in iron is here calculated by means of the Hillert-Jarl phenomenological model [77]. According to this model, $H^{\mathrm{mag}}(T)$ is expressed by

$$
H^{\mathrm{mag}}(T)=R T_{\mathrm{C}} \ln (B+1) f(\tau),
$$

where $R$ is the gas constant, $T_{\mathrm{C}}=1043 \mathrm{~K}$ the Curie temperature in iron [58], $B$ the magnetic moment (2.22 Bohr magnetons in iron [58]), and $\tau=T / T_{\mathrm{C}}$. The function $f(\tau)$ has two different expressions in the FM and PM regions, respectively:

$$
f(\tau)= \begin{cases}\frac{1}{A}\left[\frac{79}{140 P}-\frac{474}{497}\left(\frac{1-P}{P}\right)\left(\frac{\tau^{4}}{2}+\frac{\tau^{10}}{15}+\frac{\tau^{16}}{40}\right)\right], & \text { if } \tau<1 \\ \frac{1}{A}\left[\frac{\tau^{-4}}{2}+\frac{\tau^{-14}}{21}+\frac{\tau^{-24}}{60}\right], & \text { if } \tau \geqslant 1\end{cases}
$$

$P$ is a geometry factor equal to 0.40 for bcc crystals, whereas the parameter $A$ is equal to

$$
A=\frac{518}{1125}+\frac{11692}{15975}\left(\frac{1-P}{P}\right) \text {. }
$$


Finally, the factor $H(T)$ appearing in Eq. (8) is normalized with respect to the magnetic excess enthalpy at $0 \mathrm{~K}$, which can be obtained by setting $\tau=0$ :

$$
H^{\mathrm{mag}}(0)=R T_{\mathrm{C}} \ln (B+1) \frac{1}{A} \frac{79}{140 P} .
$$

[1] S. C. Glade, B. D. Wirth, G. R. Odette, and P. Asoka-Kumar, J. Nucl. Mater. 351, 197 (2006).

[2] M. Hernández-Mayoral and D. Gómez-Briceño, J. Nucl. Mater. 399, 146 (2010).

[3] E. Meslin, B. Radiguet, and M. Loyer-Prost, Acta Mater. 61, 6246 (2013).

[4] Y. Nishiyama, K. Onizawa, M. Suzuki, J. W. Anderegg, Y. Nagai, T. Toyama, M. Hasegawa, and J. Kameda, Acta Mater. 56, 4510 (2008).

[5] J. P. Wharry and G. S. Was, J. Nucl. Mater. 442, 7 (2013).

[6] T. Toyama, Y. Nagai, A. Almazouzi, M. Hatakeyama, M. Hasegawa, T. Ohkubo, E. Van Walle, and R. Gerard, Mater. Trans. 54, 2119 (2013).

[7] G. R. Odette and R. K. Nanstad, JOM: J. Min. Met. Mater. Sci. 61, 17 (2009).

[8] M. K. Miller, K. A. Powers, R. K. Nanstad, and P. Efsing, J. Nucl. Mater. 437, 107 (2013).

[9] D. Terentyev, L. Malerba, and A. V. Barashev, Philos. Mag. Lett. 85, 587 (2005).

[10] M. Lambrecht and A. Almazouzi, J. Nucl. Mater. 385, 334 (2009).

[11] K. Yabuuchi, M. Saito, R. Kasada, and A. Kimura, J. Nucl. Mater. 414, 498 (2011).

[12] A. R. Allnatt and A. B. Lidiard, Atomic Transport in Solids (Cambridge University Press, Cambridge, 2003).

[13] T. Garnier, M. Nastar, P. Bellon, and D. R. Trinkle, Phys. Rev. B 88, 134201 (2013).

[14] T. Garnier, V. R. Manga, D. R. Trinkle, M. Nastar, and P. Bellon, Phys. Rev. B 88, 134108 (2013).

[15] L. Messina, M. Nastar, T. Garnier, C. Domain, and P. Olsson, Phys. Rev. B 90, 104203 (2014).

[16] M. Nastar, Philos. Mag. 85, 3767 (2005).

[17] A. D. Le Claire, in Physical Chemistry: An Advanced Treatise, edited by H. Eyring (Academic, New York, 1970).

[18] P. Y. Serruys and G. Brebec, Philos. Mag. A 46, 661 (1982).

[19] Y. Okamura and A. R. Allnatt, J. Phys. C: Solid State Phys. 16, 1841 (1983).

[20] T. Garnier, D. R. Trinkle, M. Nastar, and P. Bellon, Phys. Rev. B 89, 144202 (2014).

[21] C. Domain and C. S. Becquart, Phys. Rev. B 71, 214109 (2005).

[22] F. Soisson and C.-C. Fu, Phys. Rev. B 76, 214102 (2007).

[23] H. Amara, C.-C. Fu, F. Soisson, and P. Maugis, Phys. Rev. B 81, 174101 (2010).

[24] S. Huang, D. L. Worthington, M. Asta, V. Ozolins, G. Ghosh, and P. K. Liaw, Acta Mater. 58, 1982 (2010).

[25] S. Choudhury, L. Barnard, J. D. Tucker, T. R. Allen, B. D. Wirth, M. Asta, and D. Morgan, J. Nucl. Mater. 411, 1 (2011).

[26] P. Olsson, T. P. C. Klaver, and C. Domain, Phys. Rev. B 81, 054102 (2010).
[27] C. S. Becquart and C. Domain, Curr. Opin. Solid State Mater. Sci. 16, 115 (2012).

[28] M. Nastar and F. Willaime, Phys. Rev. B 51, 6896 (1995).

[29] L. Ventelon, F. Willaime, C.-C. Fu, M. Heran, and I. Ginoux, J. Nucl. Mater. 425, 16 (2012).

[30] A. Janotti, M. Krčmar, C. L. Fu, and R. C. Reed, Phys. Rev. Lett. 92, 085901 (2004).

[31] M. Krčmar, C. Fu, A. Janotti, and R. C. Reed, Acta Mater. 53, 2369 (2005).

[32] T. Toyama, F. Takahama, A. Kuramoto, H. Takamizawa, Y. Nozawa, N. Ebisawa, M. Shimodaira, Y. Shimizu, K. Inoue, and Y. Nagai, Scr. Mater. 83, 5 (2014)

[33] M. Nastar, Phys. Rev. B 90, 144101 (2014).

[34] M. Nastar, Philos. Mag. 85, 641 (2005).

[35] M. Nastar and F. Soisson, Comprehensive Nuclear Materials (Elsevier, New York, 2012), Chap. 1.18, pp. 471-496.

[36] A. D. Le Claire, J. Nucl. Mater. 69-70, 70 (1978).

[37] N. Sandberg, Z. Chang, L. Messina, P. Olsson, and P. Korzhavyi, Phys. Rev. B 92, 184102 (2015).

[38] B. Jönsson, Z. Metallkd. 83, 349 (1992).

[39] G. Kresse and J. Hafner, Phys. Rev. B 47, 558 (1993).

[40] G. Kresse and J. Hafner, J. Phys.: Condens. Matter 6, 8245 (1994).

[41] G. Kresse and J. Hafner, Phys. Rev. B 49, 14251 (1994).

[42] P. E. Blöchl, Phys. Rev. B 50, 17953 (1994).

[43] G. Kresse and D. Joubert, Phys. Rev. B 59, 1758 (1999).

[44] J. P. Perdew, K. Burke, and Y. Wang, Phys. Rev. B 54, 16533 (1996).

[45] G. Mills, H. Jónsson, and G. K. Schenter, Surf. Sci. 324, 305 (1995).

[46] H. Jónsson, G. Mills, and K. W. Jacobsen, in Classical and Quantum Dynamics in Condensed Phase Simulations, edited by B. J. Berne, G. Ciccotti, and D. F. Coker (World Scientific, Singapore, 1998).

[47] G. Henkelman, B. P. Uberuaga, and H. Jónsson, J. Chem. Phys. 113, 9901 (2000).

[48] Y. Mishin, M. R. Sørensen, and A. F. Voter, Philos. Mag. A 81, 2591 (2001).

[49] G. H. Vineyard, J. Phys. Chem. Solids 3, 121 (1957).

[50] G. Lucas and R. Schäublin, Nucl. Instrum. Methods Phys. Res., Sect. B 267, 3009 (2009).

[51] R. D. Hatcher, R. Zeller, and P. H. Dederichs, Phys. Rev. B 19, 5083 (1979).

[52] S. S. Pohlong and P. N. Ram, J. Phys.: Condens. Matter 10, 10901 (1998)

[53] J. Wallenius, P. Olsson, C. Lagerstedt, N. Sandberg, R. Chakarova, and V. Pontikis, Phys. Rev. B 69, 094103 (2004).

[54] A. Seeger, Phys. Status Solidi A 167, 289 (1998).

[55] M.-C. Marinica, M. Athènes, and F. Willaime, High temperature properties of vacancy and interstitial type defects in tungsten, Technical Report No. WP13-MAT-01-IREMEV-01-03, EFDA, 2014. 
[56] T. Ohnuma, N. Soneda, and M. Iwasawa, Acta Mater. 57, 5947 (2009).

[57] O. I. Gorbatov, P. A. Korzhavyi, A. V. Ruban, B. Johansson, and Y. N. Gornostyrev, J. Nucl. Mater. 419, 248 (2011).

[58] C. Kittel, Introduction to Solid State Physics, 8th ed. (Wiley, New York, 2004).

[59] F. Willaime, A. Satta, and M. Nastar, Int. J. Quantum Chem. 77, 927 (2000)

[60] N. Sandberg and R. Holmestad, Phys. Rev. B 73, 014108 (2006).

[61] A. Claisse and P. Olsson, Nucl. Instrum. Methods Phys. Res., Sect. B 303, 18 (2013).

[62] W. von Meyer and H. Neldel, Z. Tech. Phys. 18, 588 (1937).

[63] A. Yelon, B. Movaghar, and R. S. Crandall, Rep. Prog. Phys. 69, 1145 (2006).

[64] G. Boisvert, L. J. Lewis, and A. Yelon, Phys. Rev. Lett. 75, 469 (1995).

[65] M.-C. Marinica, C. Barreteau, D. Spanjaard, and M.-C. Desjonquères, Phys. Rev. B 72, 115402 (2005).

[66] D. Emin, Phys. Rev. Lett. 32, 303 (1974).

[67] A. Yelon, B. Movaghar, and H. M. Branz, Phys. Rev. B 46, 12244 (1992).

[68] P. B. Wells, T. Yamamoto, B. Miller, T. Milot, J. Cole, Y. Wu, and G. R. Odette, Acta Mater. 80, 205 (2014).

[69] E. Meslin, M. Lambrecht, M. Hernández-Mayoral, F. Bergner, L. Malerba, P. Pareige, B. Radiguet, A. Barbu, D. GómezBriceño, A. Ulbricht, and A. Almazouzi, J. Nucl. Mater. 406, 73 (2010).
[70] B. Radiguet, S. Cammelli, E. Meslin, M. Hernández-Mayoral, and P. Pareige (private communication).

[71] M. K. Miller, K. F. Russell, M. A. Sokolov, and R. K. Nanstad, J. Nucl. Mater. 361, 248 (2007).

[72] Y. Nagai, K. Takadate, Z. Tang, H. Ohkubo, H. Sunaga, H. Takizawa, and M. Hasegawa, Phys. Rev. B 67, 224202 (2003).

[73] See Supplemental Material at http://link.aps.org/supplemental/ 10.1103/PhysRevB.93.184302 for detailed figures and tables reporting, for each impurity in iron: the solute correlation factor as a function of temperature, the solute diffusion coefficient in the range $800-1800 \mathrm{~K}$, and the fitted activation energies and prefactors in the range 400-800 K. In addition, the reader can find the correcting factors to be applied to solute diffusion coefficients at any temperature, to account for the mismatch with experimental values.

[74] J. R. Manning, Phys. Rev. B 4, 1111 (1971).

[75] H. Bakker, H. P. Bonzel, C. M. Bruff, M. A. Dayananda, W. Gust, J. Horváth, I. Kaur, G. V. Kidson, A. D. Le Claire, H. Mehrer, G. E. Murch, G. Neumann, N. Stolica, and N. A. Stolwijk, in Diffusion in Solid Metals and Alloys, Landolt-Börnstein, New Series, Group III, Vol. 26, edited by H. Mehrer (Springer, Berlin, 1990).

[76] G. Neumann and C. Tuijm, Self-Diffusion and Impurity Diffusion in Pure Metals: Handbook of Experimental Data (Elsevier, Oxford, 2011).

[77] M. Hillert and M. Jarl, CALPHAD: Comput. Coupling Phase Diagrams Thermochem. 2, 227 (1978). 